\title{
Use of cultivated plants and non-plant remedies for human and animal home- medication in Liubań district, Belarus
}

Renata Sõukand ${ }^{1,2^{*}}$ D, Yanina Hrynevich ${ }^{3}$, Julia Prakofjewa ${ }^{3}$, Tatsiana Valodzina ${ }^{3}$, Iryna Vasilyeva ${ }^{3}$, Jury Paciupa ${ }^{3}$, Aliaksandra Shrubok ${ }^{3}$, Aliaksei Hlushko ${ }^{3}$, Yana Knureva $^{3}$, Yulia Litvinava ${ }^{4}$, Siarhei Vyskvarka ${ }^{5}$, Hanna Silivonchyk ${ }^{6}$, Alena Paulava ${ }^{3}$, Mare Kõiva ${ }^{2}$ and Raivo Kalle ${ }^{2,7}$

\begin{abstract}
Background: To use any domestic remedy, specific knowledge and skills are required. Simple logic dictates that the use of wild plants in the context of limited interaction with nature requires prior identification, while in the case of non-plant remedies and cultivated plants this step can be omitted. This paper aims to document the current and past uses of non-plant remedies and cultivated plants in the study region for human/animal medication; to analyze the human medicinal and veterinary use areas in the context of the remedy groups; to qualitatively compare the results with relevant historical publications; and to compare the intensity and purpose of use between the remedy groups.
\end{abstract}

Methods: During field studies 134 semi-structured interviews were conducted with locals from 11 villages in the Liubań district of Belarus. Currently used home-remedies as well as those used in the past were documented by employing the folk history method. The subject was approached through health-related uses, not by way of remedies. Interview records were digitalized and structured in Detailed Use Records in order to ascertain local perceptions. An Informant Consensus Factor (FIC) was calculated for remedy groups as well as for different use categories.

Results: In the human medication area the use of nearby remedies was neither very diverse nor numerous: 266 DUR for 45 taxa belonging to 27 families were recorded for cultivated plants along with 188 DUR for 58 different non-plant remedies. The FIC values for both remedy groups were lower than for wild plants. In the ethnoveterinary medicine use area there were 48 DUR referring to the use of 14 cultivated plant taxa from 12 families and 72 DUR referring to the use of 31 non-plant remedies. The FIC value for the whole veterinary use area of cultivated plants was relatively low, yet similar to the FIC of wild plants.

Conclusions: Differences between remedy groups were pronounced, indicating that in domestic human medicine cultivated plants and non-plant remedies are either remarkably less important than wild ones or not considered worth talking about. In ethnoveterinary medicine non-plant remedies are almost equally important as wild plants, while cultivated plants are the least used. People in study area seem to still more often rely on, or are more willing to talk to strangers about, wild plants, as promoted by both official medicine and popular literature.

Keywords: Belarus, Non-plant remedies, Cultivated plants, Wild plants, Medicinal plants, Local knowledge, Ethnobotany, Ethnopharmacology, Ethnoveterinary, Liubań

\footnotetext{
* Correspondence: renata.soukand@unive.it; renata.herba@gmail.com

${ }^{1}$ Department of Environmental Sciences, Informatics and Statistics, Università

Ca' Foscari Venezia, via Torino 155, 30172 Mestre, VE, Italy

${ }^{2}$ Estonian Literary Museum, Vanemuise 42, 51003 Tartu, Estonia

Full list of author information is available at the end of the article
} 


\section{Background}

Academic human and veterinary medicine has advanced rapidly during the last few centuries. Educated help has become very effective, widespread, and rather affordable (or even free) in most European countries, making academic medicine the first and often only choice for the majority of Europeans. Yet, complementary and alternative medicine remains popular and is used for treating specific alignments [1]. Herbal medicines are often highlighted as the most popular among different treatments (for example [2]).

Quite logically, most researched remedies in the enthobotanical literature appear to be plants, leaving aside other possible means used for healing in the home setting. Research on the traditional or local medicinal (and very rarely veterinary) use of plants is quite often paired with the documentation of the food-use of (wild) plants [3, 4], while non-plant remedies are rarely documented, and if they are they concentrate on only a single remedy (such as [5]). Yet, recent ethnobotanical research has shown that in Eastern Europe a wide variety of nonplant remedies are still used for healing different human and (more rarely) animal health conditions [6].

In order to use any domestic remedy, specific knowledge and skills regarding what remedy to use to treat a specific disease and how to make it, as well as how to apply or preserve it, are required. For wild plants that grow outside of direct reach, an additional set of specific knowledge is needed, including where they can be found and how to recognize or differentiate them from similar taxa. Hence, the use of non-plant remedies requires a less detailed knowledge set compared to the one needed for the use of plants.

Cultivated plants fall somewhere in between wild plants and non-plant remedies in terms of availablity at home: they grow in close proximity to people, and hence are more or less at hand, yet one has to possess specific knowledge regarding their cultivation. Moreover, through cultivation the taxa are well known, and as a result the identificaton step needed for wild taxa can be omitted. A study examining changes in medicinal plant use in Estonia over the course of a century suggested that the use of plants tended by humans has increased over time as people have fewer encounters with nature and they feel more confident using plants they know for certain [7]. This suggests that the use of cultivated plants could also now be preferred or at least as popular as the use of wild ones, as the historical legacy is quite similar to that in Estonia. Yet, in Belarus, no specific studies of medicinal plants cultivated in home gardens have been conducted. However, some additional data apart from a list of the most common cultivated plants can be found in the ethnographic literature. For example, the most diverse collection of archival data from the Polish-
Lithuanian-Belarusian borderland was collected in the 1930s by a Polish ethnographer from Lviv, Adam Fischer. These data were originally gathered for publication in the first part of the Lexicon of Slavic beliefs and customs, dedicated to plant uses in traditional Slavonic culture, but this objective has not yet been realized. Modern Polish researchers have analyzed and published some archival data [8]. In Fischer's archival data home garden plants are represented by 18, mainly ornamental, species: peony (Peonia sp.), marigold (Calendula officinalis), Tagetes spp., mallow (Malva sp.), garden nasturtium (Tropaeolum majus), perennial phlox (Phlox paniculata), panicled aster (Symphyotrichum lanceolatum) and sneezewort (Achillea ptarmica). Two species were mentioned as potted plants, namely agave (Agave sp.) and surprisingly dwarf everlast (Helichrysum arenarium). Gourd (Lagenaria siceraria) was planted for its fruits, which then were used indoors - mounted on wardrobes and tile stoves for ornamental purposes. This group comprises valuable and useful plants which could simultaneously be treated as ornamental and medicinal: mint (Mentha spp.), rue (Ruta graveolens), poppy (Papaver sp.), elecampane (Inula helenium) and southernwood (Artemisia abrotanum) [8].

In the post-war period since the 1960s the use of cultivated medicinal plants has become the subject of study in pharmacology as well as agricultural and veterinary sciences, for example $([9,10]$, etc). In the 1990 s and 2000 s, brochures and encyclopedias on the use of cultivated plants from home gardens for medicinal purposes gained in popularity, for instance $([11,12]$, etc).

During ethnobotanical and ethnomedicinal fieldwork conducted in the Liubań (Аюбань) district of Belarus we documented, along with the use of wild plants (outlined in [13]), the use of all non-plant remedies pertaining to humans and animals as well as the medicinal and veterinary use of cultivated plants. Initial analysis of the results revealed that the uses of both remedy groups are quite limited, in both nomenclature and diversity of use, compared to wild food plants. However, the number of interviewees and depth of the interviews allow for deeper insight. Moreover, as the historical literature on Belarus covers non-plant remedies to a greater extent than the use of medicinal plants, it is a good opportunity to determine if recorded historical uses of non-plant remedies are still practiced or at least remembered.

As similar studies rarely assess the use of non-plant remedies, we decided to examine the subject and compare the intensity and diversity of use of different kinds of remedy groups - in this case non-plant remedies as well as cultivated and wild plants (the last group was covered in [13]) - to establish if there are any differences in use-patterns within or between the groups. With this information we aim to establish if virtual proximity to 
the domestic arena and diverse practical availability influence the importance of a remedy in domestic human and veterinary medicine.

The specific aims of this study are: 1) to document the current and past uses of non-plant remedies and cultivated plants in the Liubań district of Belarus for human/ animal medication; 2) to analyze the human medicinal and veterinary use areas in the context of non-plant remedies and cultivated plants; 3) to qualitatively compare the results with relevant historical publications regarding non-plant remedies and cultivated plants in Belarus; and 4) to compare the intensity and purpose of use between three remedy groups: non-plant, cultivated plants and wild plants.

\section{Data and methods}

\section{Subjects of analysis}

To examine the idea of the importance of the perceptional distance of primary ingredients to the domestic arena, two nearby remedy groups were thoroughly assessed:

Cultivated plants: this encompassed all plants that were intentionally cultivated in order to be eaten (at least some part of them), even if they run wild (like many Lamiaceae species) or are native and grow wild, but are, for medicinal reasons, collected mainly from cultivated specimens (like two Ribes species). This group also included self-planted tobaco (Nicotiana spp.), bedding plants (like Paeonia spp.), potted plants (like Pelargonium spp. or Callisia fragrans), and plants obtained from outside the household (store-bought) and clearly of foreign origin (Piper nigrum, Camellia sinensis). The main criteria for identifying plant as "cultivated" was that it was either grown by the person themself or obtained in a "ready to use form" so that no special identification was needed.

Non-plant remedies: this comprised those parts of plants not permitting or not requiring, from the interviewee's perspective, identification of the taxon (like chaff); products made from plants (such as bread, bread mold, vodka, food oil of any origin, etc.) given that the specific species used for their preparation are not mentioned by the interviewee; all animal parts (fat, meat) and products (milk, honey); as well as all other medicaments not purchased from a pharmacy for medicinal purposes nor prescribed by a doctor.

\section{Region}

Belarus is located between Lithuania, Latvia, Russia, Ukraine and Poland, in Central and Eastern Europe. It covers a territory of 207.6 thousand sq. $\mathrm{km}$. The climate is moderately continental, transitional between maritime and continental, as it is positioned in the temperate latitudes of the western part of the East European Plain [14]. The Chernobyl disaster, which happened 30 years ago, affected Belarus more than any other country, causing high radioactivity and extensive relocation of people.

Temporal changes in home-gardening in Belarus.

Until the eighteenth century the main vegetable crops grown in the Belarusian lands were onions, cucumbers, cabbage, beets, turnips, carrots, poppy, garlic, parsley, parsnips, and hops, among others. During the second half of the eighteenth century on manor farms people began to plant potatoes, strawberries, beans, cauliflower, swede and spinach. Gardening was poorly developed and apples, plums, gooseberries, and currants were primarily grown. In aristocratic parks and greenhouses, however, rare species of fruit trees and shrubs, such as oranges, peaches, grapes, etc. [15], were planted. The lack of necessary data in the sources of the eighteenth century does not allow determining the place occupied by vegetable growing and gardening in the life of Belarusian peasants [16].

In the nineteenth century vast acres of land were occupied by the cultivation of lentils, beans, and peas [17]. In the Minsk region hops, mustard, coriander, melons and pumpkins, mint, radishes, onion and garlic, lettuce, parsley, poppy, asparagus and tobacco were actively planted [18]. During this period gardens occupied a peripheral place in the economic life of the peasantry [19]. Home-gardens were located close to the house, merging it with housekeeping, and so the garden was almost under sole control of women [20]. The small size of the yard limited the range of cultivated crops to only the most essential ones [19]. At the end of the nineteenth century most of the yard was occupied by hemp and barley, which, according to peasants, were more useful than vegetables [21]. Hemp was cultivated for its fiber, seeds and oil, as well as for medical purposes. At the end of the nineteenth century the cultivation of hemp was one of the basic sources of income of peasants in the provinces of Mahileu and, in part, Minsk [22]. Barley was cultivated as a cereal crop for food, brewing material and animal fodder [23].The most common cultivated plants included cabbage, cucumbers, onion and beets. Occasionally pumpkins, radishes, turnips and beans were also grown [21]. Kidney beans were largely unknown to peasants in the nineteenth century; and mixed crops were a distinctive feature of peasant gardens [21]. Peasant women from suburban villages grew leafy vegetables, such as lettuce, green onion, dill, sorrel, parsley, spinach, etc., which were sold in nearby towns [24, 25]. But above all, people engaged in horticulture to provide for the needs of the family [26]. The cultivation of early vegetables in greenhouses was still not widespread in the nineteenth century [21]. The traditional fertilizer was cattle manure [26].

The twentieth century brought along changes in the structure of the workforce division in villages; however, 
home-gardening remained one of the primary means of acquiring food for local inhabitants, and even now horticulture remains one of the most important agricultural practices in Belarus (on farms and estates of burghers, village dwellers and even summer residents).

In the researched region literally everyone in the village able to hold a spade keeps a home-garden, which is a relatively small plot close to the house. Most of the space in such gardens is covered by vegetables and fruiting trees, while flower gardens are worth mentioning only when talking about bigger villages. This is quite understandable as the choice of food available in shops is rather limited and is not oriented toward vegetables or fresh food. However, retail shops are present in larger villages and inhabitants of smaller villages are supplied by mobile shops that visit twice a week. Moreover, food is relatively expensive and collective farm workers do not earn much money. For these reasons many villagers still keep domestic animals (pigs, goats, ducks, hens, cows and horses), although admittedly their numbers have diminished during the last several years.

\section{Data collection}

The portion of the data contributing to this paper was obtained during a wider ethnomedicinal field study conducted in 11 villages of the village council of Asaviec, Liubań district (Fig. 1) in May 2016 as a practical part of a development cooperation project financed by the Ministry of Foreign Affairs of Estonia. The same 134 interviews with locals (born in the villages where the interviews took place or elsewhere in the district of Lubań), as in [13], were selected for this analysis. About two-thirds of the interviewees were women and onethird men, which was due to the low representation of elderly men in the villages; the mean age of the interviewees was 63 years, while the oldest interviewee was 92 years old and the youngest was 27 years old. For more information on the researched area and further details of the study see [13].

This research documented currently used homeremedies as well as those used in the past by employing the folk history method, sensu [27] (reconstruction of historical events through the memory of common people). The subject was approached through specific uses, not by way of remedies. The first part of the interview concerned the use of wild plants and local foods, and this may have introduced little bias as some people paid more attention to wild plants. Yet, in the second part of the interview the domestic sphere of healing was approached through specific illnesses, emphasizing that

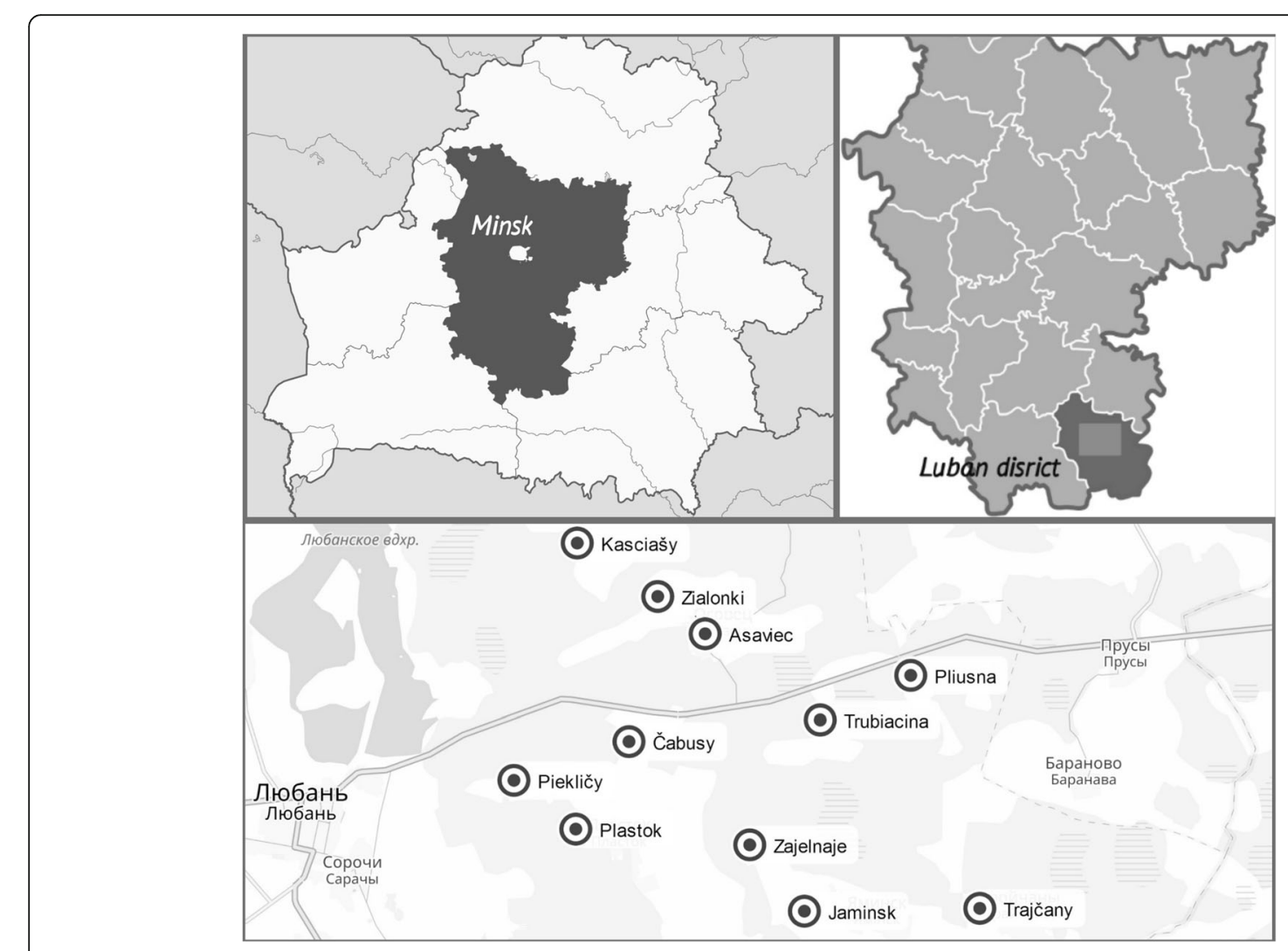

Fig. 1 Field research site 
researchers were interested in all possible means used for healing. In the semistructured format, interviewees were asked to recall what remedies were used to treat most common disease categories using layperson terminology, such as respiratory and cold-related diseases (cold, sore throat, rhinitis, earache, etc.), neurological diseases (headache, nervousness, etc.), cardiovascular ailments (heart problems, blood pressure, etc.) gastrointestinal complaints (stomach ache, diarrhea), musculoskeletal disorders (foot ache, rheumatic diseases, etc.), dermatological conditions (wounds, burns, cuts, skin diseases, etc.), ophthalmological issues (improving vision, eye diseases), oral and dental care (aching tooth, mouth diseases), nephrological ailments (kidney diseases), infection and uses related to other health concerns and wellbeing (prophylactics, being healthy) as well as cosmetic issues (hair and skin care, disinfection, good scent, etc.). After the interviewee responded, a control question was asked, namely if he or she could recall any other specific diseases and their treatment. The veterinary question was addressed more broadly (when animals get ill or how to keep them healthy). Interviewees were allowed to elaborate on the subject as long as they stayed within the health-remedy framework. The interviewees were asked to state when they have used the named remedies and if they have used them personally.

Interviews were voice-recorded upon permission of the interviewee; field notes were also taken and in the majority of cases names recorded, according to the rules prescribed by the local institution. The purpose of the study was explained to each person and prior informed consent was obtained from all interviewees. The Code of Ethics of the International Society of Ethnobiology [28] was followed. All interviews were then transcribed and anonymized before further analysis. The original voicerecorded interviews as well as their transcripts are stored at the The Center for Belarusian Culture, Language and Literature Research within the Archives of The Institute of Art, Ethnography and Folklore, named after K. Krapiva (AIAEF 23-16-2). Anonymized copies of interview transcripts are also stored at the Scientific Archive of the Estonian Folklore Institute (EFISA Valgevene2016) located in the Estonian Literary Museum.

General ethnobotanical practice does not recommend collecting cultivated plant vouchers, therefore all the plants were identified on the spot and only a limited number of voucher specimens were taken to ensure precise identification; in some cases, where people willingly shared their stores, dried plant samples were also archived. Collected voucher specimens were dried and identified with the help of Toomas Kukk (Curator of the Estonian University of Life Sciences herbaria); vouchers are deposited at the Estonian University of Life Sciences herbaria (TAA), assigned herbarium numbers within the range TAA0132555-0132710, and also bearing numbers LJUB001-152. Dried plant samples collected from respondents are deposited at the Scientific Archive of the Estonian Folklore Institute (EFISA Valgevene2016, bearing numbers LJUD001-085). Taxonomic identification, botanical nomenclature, and family assignments of cultivated plants followed the Flora Europaea [29], The Plant List database [30], and the Angiosperm Phylogeny Group IV [31].

Non-plant remedies were identified on the basis of the name used by the interviewees, which as a rule was sufficient for precise identification. Only in few cases were some additional clarification questions asked.

\section{Data analysis}

Anonymized records were entered into a Microsoft Excel spread sheet. As with wild plants [13], to follow emic categories, information was structured in Detailed Use Records (DUR adopted from [32]), where interviewees $(i)$ mention a specific use $(u$, e.g. emic disease/ illness category [cough, sore throat, heart disease, back pain, etc.], emic veterinary treatment) of a remedy or plant part ( $p$, e.g. fruits, leaves, aerial parts, flowers, etc.) prepared in a certain way $(w$, e.g. topical application of the remedy, tea [plants macerated in hot water], decoction [plants boiled in water], tincture [plants or other substances macerated in alcohol - either applied or drunk], special preparation, etc.). Interviewee-defined emic categories were employed to ascertain local perceptions.

For every taxon, the number of Use Citations (UC number of people who claimed the (specific) use of the remedy during the interview) and the number of DUR were calculated for the sum of all uses and separately for medicinal and veterinary areas. For general and emic disease/illness categories, Use Instance (UI), representing the attribution of one specific taxon to a disease category, regardless of the number of people mentioning the specific use, was calculated. Following the recommendation given in several recent publications [33, 34] and to illustrate the diversity of various uses, uses mentioned by only one person were also included.

An Informant Consensus Factor (FIC [35]) was calculated for both remedy groups (cultivated plants and nonplant remedies) as well as for different use categories within each of the groups.

$\mathrm{FIC}=\mathrm{nUC}-\mathrm{nT} /(\mathrm{Nuc}-1)$.

$\mathrm{nUC}=$ number of use citations for a particular plant-

use category;

$\mathrm{nT}=$ number of taxa or species that are used for that plant use category. 
FIC values range between 0 and 1 , where ' 1 ' indicates the highest level of informant consent.

The reliability criterion [36] was also assessed to detect the proportion of remedies used by more than three people.

On a temporal scale all uses were divided into four blocks: 1) continuously used (reported to be used throughout life), 2) past uses (uses that were talked about in the past tense), 3) temporary uses (practiced for the limited period of time during adulthood), and 4) adulthood uses (practices that were not known to the person during childhood, but picked up at some point in adulthood and still practiced). The issue of the temporal scale bears some limitations as not all people responded to the question regarding the time in which a remedy was used in an unambiguously interpretable way (and at times the interviewers were too engrossed in the conversation to ask clarifying questions). For about one-third of the use reports, attribution to the temporal block was made on the basis of context (verb tense the person used, time period they were talking about, etc.) and, therefore, this scale reflects in part the approach of the researcher. However, as all researchers collecting data asked the same questions about the same diseases treated, even a slightly vague interpretation of the temporal scale deserves to be analyzed.

\section{Qualitative comparison with historical uses and quantitative comparison with medicinal and ethnoveterinary uses of wild plants}

The peak of research on the ethnomedicine of Belarus occurred at the end of the nineteenth century [36].

In those publications special attention was paid to the causes of illnesses, their prevention, and ways of ritual treatment, but the comments were developed in accordance with the attitudes of the collectors - i.e. to show the "downtrodden" peasants or romanticize their "antiquity". Representatives of the mythological school enthusiastically talked about the "ghosts and demons" of diseases and ways of communicating with them [18, 26, $37,38]$. By the middle of the nineteenth century, among the collectors and researchers of folk customs, there was already the idea of a separate sphere of national knowledge - "folk medicine". Materials on folk medicine were systematically collected and discussed in publications [37-46]. Folk medicine can be understood from two different perspectives: 1) to reveal the reasons for the stability of folk techniques and 2) to enrich medical science with unknown medicines. The common tendency in evaluating traditional medicinal practices was to consider "superstitious" techniques (from the collectors' point of view) as "age-old backwardness" and "useful" ones as "folk wisdom and experience".
Special attention was also paid to the study of medicinal plants. At the end of nineteenth century ethnographers encouraged the creation of herbariums of local flora. For example, E. Orzeszkowa established a full herbarium of the vegetation of the Neman river region, in which the specimens were accompanied by a description of their uses in folk medicine, as well as related proverbs and beliefs [47]. The historical sources also allow us to see a more diverse picture of the cultivated plants and non-plant remedies employed in ethnoveterinary medicine in the 19th and early 20th centuries [48-56].

The historical vicissitudes of the twentieth century and changes in the scholarly approach to ethnomedicine $[57,58]$ did not allow for the gathering of much comparative data from that period.

In Soviet times there were almost no folkloric or ethnographic studies on the subject of folk medicine, which seems to indicate the absence of official support for such documentation at that time. At the end of the twentieth century publicatons focused on the ethnocultural component of folk healing and the mythology of diseases. The first volume of the "Polack Ethnographic Collection" (Issue 1, "Folk Medicine of Belarusians from Padzvinnie region") [59] was dedicated to folk medicine. Summarizing the results of a study was the volume "Traditional medicine and ritual-magic practices" prepared by T.Valodzina from the series "Belarusian Folk Art" [37], which presents a chronologically blurred section of Belarusian magical healing. This volume is a complete compendium of folk medicinal ritual-magical practices, collected from a variety of printed sources and archives. A large part of the material was collected by the author during more than 100 expeditions between 1993 and 2010 to all regions of Belarus under a special program (more than 7000 folklore units were recorded).

As of today, Polish scientists have reviewed the unpublished sources of the late nineteenth century on ethnobotany, including the Belarusian lands: a questionnaire by Yu. Rostafinski (1883) [60] and a manuscript by $\mathrm{M}$. Federowski, who structured his data in accordance with the earlier questionnaire of Rostafinski [61].

Therefore, qualitative comparisons with the current collected data and historical records covering all of Belarus can be drawn for both human and animal medication, especially when it concerns non-plant remedies.

The results obtained for cultivated plants and remedies were compared with similar results for wild plants as in [13], and a few additional aspects (like the quantification of recently acquired uses) of the data regarding the use of wild food plants were further analyzed and added to the already published results. In this instance, wild plants consisted of all plants growing outside direct human cultivation, including domesticated local wild plants cultivated in home gardens, but not for food purposes 
(such as bedding plants and non-fruiting trees, which can also be encountered outside of human cultivation).

\section{Results and discussion \\ Human medication \\ Cultivated plants}

Interviewees used 45 cultivated plant taxa belonging to 27 families (Table 1). The most well represented families were Lamiaceae (six taxa), Rosaceae (five taxa), Poaceae (four taxa), Solanaceae and Asteraceae (three taxa each). The reliability criterion was met by 26 taxa (57\%).

Two hundred twenty-six DUR of cultivated plants were recorded. The six most widely utilized taxa (and named by at least $10 \%$ of interviewees) included two $\mathrm{Al}$ lium taxa (garlic A. sativum [26 DUR] and onion A. cepa [22 DUR]), followed by houseplant aloe Aloe spp. (24 DUR), potato Solanum tuberosum (20 DUR), aronia Aronia spp. (16 DUR), and cabbage Brassica oleracea (15 DUR).

Altogether $108 \mathrm{UI}$ were detected in general disease categories. The reliability criterion (at least three users) was met by 30 general UI. This group contained 19 taxa, of which only seven were used in more than one wider disease category, including garlic Allium sativum and onion Allium cepa in four disease categories, three of which (respiratory, general health and infection) were shared, while the other two were oral and dental and dermatological, respectively. Potato Solanum tuberosum was used in three general categories (dermatological, respiratory and gastrointestinal). The remaining four taxa met the reliability criterion in two disease categories: blackcurrant Ribes nigrum and black pepper Piper nigrum (respiratory and gastrointestinal in both), aloe Aloe spp. (respiratory and dermatological) and aronia Aronia spp. (gastrointestinal and cardiovascular). Among the other twelve taxa, the most well represented included ophthalmalogical use of leaves of the tea plant Camellia sinensis, gastrointestinal use of dill Anethum graveolens and Beta vulgaris, neurological use of mint Mentha spp. and cardiovascular use of Leonurus quinquelobatus, all reported by at least five people.

Individual use of cultivated plants was not very diverse. Only one person claimed to have used one taxon, namely calendula (Calendula officinalis), to treat five different illness categories, while three more taxa (Anethum graveolens, Solanum tuberosum and Mentha spp.) were used by a single individual in three different illness categories.

The Informant Consensus Factor for the entire medicinal use area was relatively low at 0.82 (251 use citations for 45 taxa). All of the general categories in the medicinal use area had lower FIC values and only five general categories had a FIC greater than 0.6.
The disease category with the highest FIC was infection (FIC 0.79 for five taxa), which was dominated by two Allium taxa and Cucurbita pepo used to treat helminthic infection. Another small category with only three taxa used was ophthalmological $(\mathrm{FIC}=0.77)$, which was dominated by Camellia sinensis (used only in this disease category to treat eye problems). The dermatological category (FIC 0.69 , ten taxa) was clearly dominated by the taxon Aloe spp. (Figure 2), which was used to treat mainly wounds, but also burns, cuts and skin inflammation. Only a few other taxa in this diease category met the reliability criterion: Allium cepa (used to treat abscess, (rotten) wounds and skin diseases), Hippophae rhamnoides (oils for treating burns), and Solanum tuberosum (for treating burns and skin diseases).

Only two taxa in the cardiovascular category (FIC $=0.64$, six taxa) met the reliability criterion: Aronia spp. to treat hypertension and Leonurus quinquelobatus to treat heart problems. The second most numerous in terms of represented taxa was the respiratory category (FIC $=0.63,18$ taxa), which consisted of six taxa, none of which were clearly dominate, that met the reliability criterion: Aloe spp. (eaten fresh against cold and macerated in alcohol or cooked to treat lung diseases or tuberculosis (in the past)), Solanum tuberosum (used as an inhalation against cold, cough and rhinitis), Allium sativum (eaten fresh, worn as a necklace to combat cold and cough, and applied topically to alleviate rhinitis), $\mathrm{Al}$ lium cepa (boiled with milk or inhaled to treat cough and rhinitis), Ribes nigrum (used as a tea against cold) and Prunus cerasus (used as a tea to combat cold and sore throat).

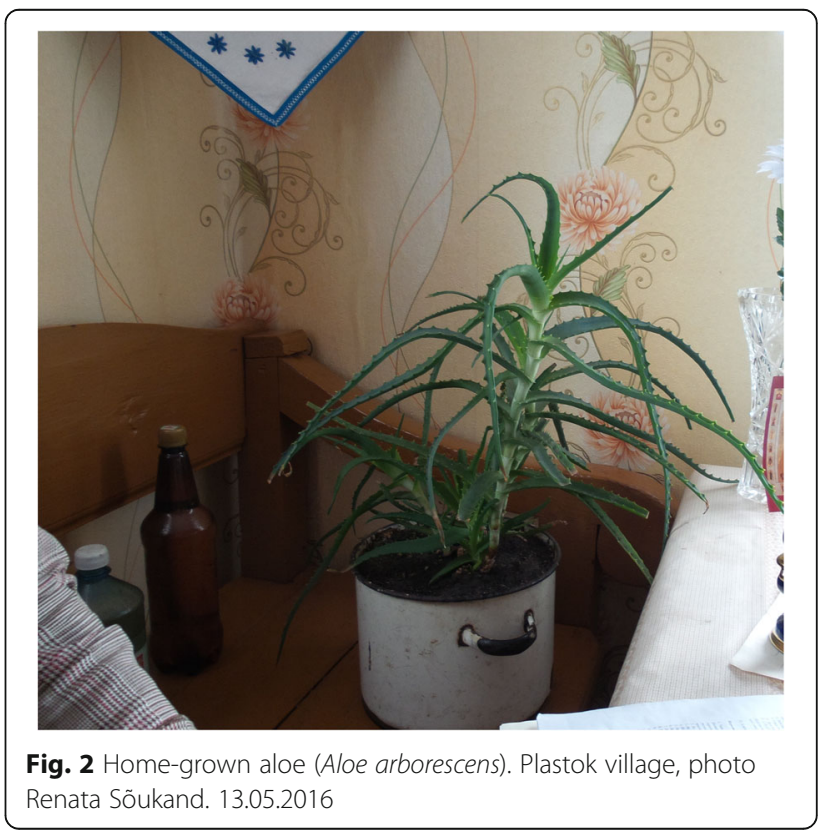


Table 1 Medicinal and ethnoveterinary uses of cultivated plants

\begin{tabular}{|c|c|c|c|c|c|}
\hline $\begin{array}{l}\text { Plant taxon, family } \\
\text { (voucher specimen code) }\end{array}$ & Local names & $\begin{array}{l}\text { Used } \\
\text { part(s) }\end{array}$ & Preparation & $\begin{array}{l}\text { Recorded medical } \\
\text { use(s) (treated disease) }\end{array}$ & UC \\
\hline \multirow[t]{4}{*}{ Beta vulgaris L., Amaranthaceae } & \multirow[t]{4}{*}{ буракі, свякла / buraki, sviakla } & \multirow[t]{3}{*}{ roots } & \multirow[t]{2}{*}{ fresh } & laxative & 3 \\
\hline & & & & constipation & 2 \\
\hline & & & $\begin{array}{l}\text { fresh, added to } \\
\text { fodder }\end{array}$ & $\begin{array}{l}\text { fodder for cows, } \\
\text { increase milk } \\
\text { production }\end{array}$ & 1 \\
\hline & & tubers & juice drunk & constipation & 1 \\
\hline \multirow[t]{13}{*}{ Allium cepa L., Amaryllidaceae } & \multirow[t]{13}{*}{ лук, цыбуля / luk, cybulia } & \multirow[t]{11}{*}{ bulbs } & baked & rhinitis & 1 \\
\hline & & & \multirow{3}{*}{$\begin{array}{l}\text { baked, topical } \\
\text { application }\end{array}$} & rotten wounds & 1 \\
\hline & & & & abscesses & 3 \\
\hline & & & & wounds & 1 \\
\hline & & & boiled with milk & cough & 1 \\
\hline & & & \multirow[t]{2}{*}{ fresh } & helmitic infection & 6 \\
\hline & & & & rhinitis & 1 \\
\hline & & & \multirow[t]{2}{*}{ inhalation } & rhinitis & 2 \\
\hline & & & & wellbeing & 1 \\
\hline & & & \multirow[t]{2}{*}{ topical applicaton } & skin diseases & 2 \\
\hline & & & & snake bites & 1 \\
\hline & & \multirow{2}{*}{$\begin{array}{l}\text { whole } \\
\text { plant }\end{array}$} & fresh & immune boosting & 1 \\
\hline & & & tea & panacea & 1 \\
\hline \multirow{16}{*}{$\begin{array}{l}\text { Allium sativum L., Amaryllidaceae } \\
\text { (LJUD037) }\end{array}$} & \multirow[t]{16}{*}{ часнык, чэснок / časnyk, česnok } & \multirow[t]{13}{*}{ bulbs } & \multirow[t]{5}{*}{ fresh } & helmitic infection & 7 \\
\hline & & & & cold & 2 \\
\hline & & & & immune boosting & 2 \\
\hline & & & & cough & 1 \\
\hline & & & & rhinitis & 1 \\
\hline & & & $\begin{array}{l}\text { alcohol } \\
\text { maceration, } \\
\text { topical application }\end{array}$ & joint pain & 1 \\
\hline & & & \multirow[t]{2}{*}{ necklace } & cold & 1 \\
\hline & & & & prophylactics & 1 \\
\hline & & & $\begin{array}{l}\text { steamed with } \\
\text { milk }\end{array}$ & helmitic infection & 1 \\
\hline & & & tea & panacea & 1 \\
\hline & & & \multirow[t]{3}{*}{ topical applicaton } & toothache & 5 \\
\hline & & & & helmitic infection & 1 \\
\hline & & & & rhinitis & 1 \\
\hline & & fruits & fresh & cancer & 1 \\
\hline & & \multirow[t]{2}{*}{ seeds } & \multirow[t]{2}{*}{ decoction } & $\begin{array}{l}\text { good for pigs } \\
\text { (vet) }\end{array}$ & 2 \\
\hline & & & & $\begin{array}{l}\text { rumination } \\
\text { problems in } \\
\text { cows (vet) }\end{array}$ & 2 \\
\hline \multirow[t]{3}{*}{$\begin{array}{l}\text { Anethum graveolens L., Apiaceae } \\
\text { (LJUD014) }\end{array}$} & \multirow[t]{3}{*}{ кроп, укроп / krop, ukrop } & \multirow[t]{3}{*}{$\begin{array}{l}\text { aerial } \\
\text { parts }\end{array}$} & boiled & $\begin{array}{l}\text { diarrhea in cows } \\
\text { and pigs (vet) }\end{array}$ & 1 \\
\hline & & & decoction & $\begin{array}{l}\text { rumination problems in } \\
\text { cows (vet) }\end{array}$ & 1 \\
\hline & & & dried & spices & 1 \\
\hline
\end{tabular}


Table 1 Medicinal and ethnoveterinary uses of cultivated plants (Continued)

\begin{tabular}{|c|c|c|c|c|c|}
\hline $\begin{array}{l}\text { Plant taxon, family } \\
\text { (voucher specimen code) }\end{array}$ & Local names & $\begin{array}{l}\text { Used } \\
\text { part(s) }\end{array}$ & Preparation & $\begin{array}{l}\text { Recorded medical } \\
\text { use(s) (treated disease) }\end{array}$ & $U C$ \\
\hline & & & fresh & spices for cucumbers & 7 \\
\hline & & & & soup & 5 \\
\hline & & & & spices & 2 \\
\hline & & & & spices for soup & 2 \\
\hline & & & & salad & 1 \\
\hline & & & & spices for sauerkraut & 1 \\
\hline & & seeds & decoction & $\begin{array}{l}\text { increase cow milk } \\
\text { production }\end{array}$ & 1 \\
\hline & & & dried & spices & 11 \\
\hline & & & & spices for meat & 4 \\
\hline & & & & spices for sausages & 3 \\
\hline & & & & spices for soup & 3 \\
\hline & & & & condiment & 1 \\
\hline & & & & spices for pork fat & 1 \\
\hline & & & & spices for potatos & 1 \\
\hline & & & & spices for salad & 1 \\
\hline & & & & flatulence & 1 \\
\hline & & & & stomachache & 1 \\
\hline & & & tea & stomachache & 4 \\
\hline & & & & burns & 1 \\
\hline & & & & flatulence in children & 1 \\
\hline \multirow[t]{2}{*}{$\begin{array}{l}\text { Petroselinum crispum (Mill.) Fuss, Apiaceae } \\
\text { (LUB134) }\end{array}$} & \multirow[t]{2}{*}{ пятрушка / piatruška } & $\begin{array}{l}\text { aerial } \\
\text { parts }\end{array}$ & fresh & men's health & 1 \\
\hline & & leaves & fresh & potency & 1 \\
\hline \multirow{4}{*}{$\begin{array}{l}\text { Albuca bracteata (Thunb.) J.C.Manning \& } \\
\text { Goldblatt, Asparagaceae }\end{array}$} & \multirow{4}{*}{$\begin{array}{l}\text { лук індзійскі, кітайская цыбуля, вазон / } \\
\text { luk indzijski, kitajskaja cybulia, vazon }\end{array}$} & \multirow[t]{4}{*}{ leaves } & \multirow{3}{*}{$\begin{array}{l}\text { alcohol } \\
\text { maceration }\end{array}$} & wellbeing & 1 \\
\hline & & & & $\begin{array}{l}\text { applied on aching } \\
\text { joints }\end{array}$ & 1 \\
\hline & & & & $\begin{array}{l}\text { applied against } \\
\text { reumatic pains }\end{array}$ & 1 \\
\hline & & & topical applicaton & burns & 2 \\
\hline \multirow{8}{*}{$\begin{array}{l}\text { Calendula officinalis L., Asteraceae } \\
\text { (LJUD032) }\end{array}$} & \multirow[t]{8}{*}{ наготкі, календула / nahotki, kaliendula } & \multirow{4}{*}{$\begin{array}{l}\text { aerial } \\
\text { parts }\end{array}$} & compress & eye problems & 1 \\
\hline & & & tea & sore throat & 1 \\
\hline & & & & stomachache & 1 \\
\hline & & & topical applicaton & cuts & 1 \\
\hline & & \multirow[t]{4}{*}{ flowers } & decoction & gingival inflamation & 1 \\
\hline & & & & hair care & 1 \\
\hline & & & $\begin{array}{l}\text { alcohol } \\
\text { maceration }\end{array}$ & heart problems & 1 \\
\hline & & & tea & stomachache & 1 \\
\hline \multirow[t]{2}{*}{ Helianthus annuus L., Asteraceae } & \multirow[t]{2}{*}{ падсолнечнік / padsolniečnik } & \multirow[t]{2}{*}{ seeds } & fresh oil & constipation & 1 \\
\hline & & & oil given & $\begin{array}{l}\text { rumination problems in } \\
\text { cows (vet) }\end{array}$ & 2 \\
\hline \multirow[t]{3}{*}{ Helianthus tuberosus L., Asteraceae } & \multirow{3}{*}{$\begin{array}{l}\text { груша земляная, тапінамбур, ціпанамбур / } \\
\text { hruša ziemlianaja, tapinambur, cipanambur }\end{array}$} & \multirow{3}{*}{$\begin{array}{l}\text { flowers } \\
\text { tubers }\end{array}$} & tea & diabetes & 1 \\
\hline & & & fresh & diabetes & 1 \\
\hline & & & fresh, fodder & $\begin{array}{l}\text { good for home } \\
\text { animals (vet) }\end{array}$ & 1 \\
\hline
\end{tabular}


Table 1 Medicinal and ethnoveterinary uses of cultivated plants (Continued)

\begin{tabular}{|c|c|c|c|c|c|}
\hline $\begin{array}{l}\text { Plant taxon, family } \\
\text { (voucher specimen code) }\end{array}$ & Local names & $\begin{array}{l}\text { Used } \\
\operatorname{part}(s)\end{array}$ & Preparation & $\begin{array}{l}\text { Recorded medical } \\
\text { use(s) (treated disease) }\end{array}$ & UC \\
\hline \multirow[t]{6}{*}{ Brassica oleracea L., Brassicaceae } & \multirow[t]{6}{*}{ капуста, капуснік / kapusta, kapusnik } & \multirow[t]{2}{*}{ brine } & feeded & $\begin{array}{l}\text { rumination problems in } \\
\text { cows (vet) }\end{array}$ & 2 \\
\hline & & & drunk & constipation & 2 \\
\hline & & \multirow[t]{4}{*}{ leaves } & \multirow[t]{4}{*}{ topical applicaton } & joint pain & 6 \\
\hline & & & & headache & 5 \\
\hline & & & & $\begin{array}{l}\text { inflamation after } \\
\text { injection }\end{array}$ & 1 \\
\hline & & & & reumatic pains & 1 \\
\hline \multirow{3}{*}{$\begin{array}{l}\text { Raphanus raphanistrum } \\
\text { subsp. sativus (L.) Domin, Brassicaceae }\end{array}$} & \multirow{3}{*}{$\begin{array}{l}\text { редзька чёрная, рэдзіска / } \\
\text { riedźka čiornaja, redziska }\end{array}$} & \multirow[t]{3}{*}{ roots } & \multirow[t]{3}{*}{ eaten fresh } & wellbeing & 1 \\
\hline & & & & kidney stones & 1 \\
\hline & & & & rhinitis & 1 \\
\hline Cannabis sativa L., Cannabaceae & канапля / kanaplia & seeds & eaten fresh & constipation & 1 \\
\hline \multirow[t]{2}{*}{$\begin{array}{l}\text { Callisia fragrans (Lindl.) Woodson, } \\
\text { Commelinaceae (LJUB153) }\end{array}$} & \multirow[t]{2}{*}{$\begin{array}{l}\text { зелёный ус, залатой ус / } \\
\text { zielionyj us, zalatoj us }\end{array}$} & \multirow[t]{2}{*}{$\begin{array}{l}\text { aerial } \\
\text { parts }\end{array}$} & $\begin{array}{l}\text { alcohol } \\
\text { maceration, } \\
\text { topical application }\end{array}$ & joint pain & 1 \\
\hline & & & tea & cancer & 1 \\
\hline \multirow{3}{*}{$\begin{array}{l}\text { Kalanchoe spp., } \\
\text { Crassulaceae }\end{array}$} & \multirow[t]{3}{*}{ каланхое / kalanchoje } & \multirow[t]{2}{*}{ leaves } & tea & sore throat & 1 \\
\hline & & & topical applicaton & wounds & 1 \\
\hline & & sap & drops into nose & rhinitis & 1 \\
\hline \multirow[t]{2}{*}{$\begin{array}{l}\text { Cucumis sativus L., } \\
\text { Cucurbitaceae }\end{array}$} & \multirow[t]{2}{*}{ агуркі / ahurki } & \multirow[t]{2}{*}{ fruits } & \multirow[t]{2}{*}{ fermented } & $\begin{array}{l}\text { rumination problems in } \\
\text { cows (vet) }\end{array}$ & 2 \\
\hline & & & & $\begin{array}{l}\text { appetizer for animals } \\
\text { (vet) }\end{array}$ & 1 \\
\hline \multirow{3}{*}{$\begin{array}{l}\text { Cucurbita pepo L., } \\
\text { Cucurbitaceae }\end{array}$} & \multirow[t]{3}{*}{ гарбуз, тыква / harbuz, tykva } & fruits & fresh & fodder for pigs (vet) & 1 \\
\hline & & seeds & dried & helmitic infection & 2 \\
\hline & & & fresh & helmitic infection & 2 \\
\hline \multirow{2}{*}{$\begin{array}{l}\text { Hippophae rhamnoides L., } \\
\text { Elaeagnaceae (LJUB126) }\end{array}$} & \multirow[t]{2}{*}{ абляпіха / abliapicha } & \multirow[t]{2}{*}{ fruits } & jam & cold & 1 \\
\hline & & & oil & burns & 3 \\
\hline \multirow{4}{*}{$\begin{array}{l}\text { Pelargonium spp., } \\
\text { Geraniaceae (LJUB112) }\end{array}$} & \multirow[t]{4}{*}{ герань / hierań } & \multirow[t]{4}{*}{ leaves } & fresh into ear & earache & 2 \\
\hline & & & inbetween cloth & moth repellent & 2 \\
\hline & & & topical applicaton & sedative & 2 \\
\hline & & & & headache & 2 \\
\hline Ribes nigrum L, Grossulariaceae & смародзіна, чорная смародзіна / & fruits & fresh & diarrhea & 1 \\
\hline & smarodzina, cornaja smarodzina & & & hypertension & 1 \\
\hline & & leaves & tea & cold & 1 \\
\hline & & & & diuretic & 1 \\
\hline & & & & wellbeing & 1 \\
\hline & & & & immune boosting & 1 \\
\hline & & & & sore throat & 1 \\
\hline & & shoots & tea & cold & 1 \\
\hline & & & & $\begin{array}{l}\text { strenghtening of } \\
\text { organism }\end{array}$ & 1 \\
\hline & & twigs & bath & diathesis in children & 1 \\
\hline & & & decoction & cold & 1 \\
\hline $\begin{array}{l}\text { Ribes uva-crispa L., } \\
\text { Grossulariaceae }\end{array}$ & агрэст, крыжоўнік / ahrest, kryžoŭnik & fruits & $\begin{array}{l}\text { alcohol } \\
\text { maceration }\end{array}$ & soporific & 1 \\
\hline
\end{tabular}


Table 1 Medicinal and ethnoveterinary uses of cultivated plants (Continued)

\begin{tabular}{|c|c|c|c|c|c|}
\hline $\begin{array}{l}\text { Plant taxon, family } \\
\text { (voucher specimen code) }\end{array}$ & Local names & $\begin{array}{l}\text { Used } \\
\text { part(s) }\end{array}$ & Preparation & $\begin{array}{l}\text { Recorded medical } \\
\text { use(s) (treated disease) }\end{array}$ & $U C$ \\
\hline $\begin{array}{l}\text { Hydrangea spp., } \\
\text { Hydrangeaceae }\end{array}$ & гартэнзія / hartenzija & leaves & topical applicaton & earache & 1 \\
\hline \multirow{2}{*}{$\begin{array}{l}\text { Hyssopus officinalis L., } \\
\text { Lamiaceae }\end{array}$} & \multirow[t]{2}{*}{ ісоп / isop } & \multirow[t]{2}{*}{ leaves } & \multirow[t]{2}{*}{ tea } & cough & 1 \\
\hline & & & & stomach problems & 1 \\
\hline \multirow[t]{7}{*}{$\begin{array}{l}\text { Leonurus quinquelobatus Gilib., Lamiaceae } \\
\text { (LJUB004) }\end{array}$} & \multirow[t]{7}{*}{ пустырнік / pustyrnik } & \multirow[t]{4}{*}{$\begin{array}{l}\text { aerial } \\
\text { parts }\end{array}$} & $\begin{array}{l}\text { alcohol } \\
\text { maceration }\end{array}$ & heart problems & 1 \\
\hline & & & decoction & heart problems & 2 \\
\hline & & & tea & sedative & 1 \\
\hline & & & & diarrhea & 1 \\
\hline & & \multirow[t]{2}{*}{ leaves } & \multirow[t]{2}{*}{ tea } & heart problems & 2 \\
\hline & & & & helmitic infection & 1 \\
\hline & & root & $\begin{array}{l}\text { alcohol } \\
\text { maceration }\end{array}$ & heart problems & 1 \\
\hline \multirow{2}{*}{$\begin{array}{l}\text { Melissa officinalis L., } \\
\text { Lamiaceae }\end{array}$} & \multirow{2}{*}{$\begin{array}{l}\text { меліса лімонная, мята лімонная, } \\
\text { лімонка, мята перачная / mielisa } \\
\text { limonnaja, miata limonnaja, limonka, } \\
\text { miata pieračnaja }\end{array}$} & \multirow[t]{2}{*}{ leaves } & \multirow[t]{2}{*}{ tea } & wellbeing & 1 \\
\hline & & & & stomachache & 1 \\
\hline \multirow{7}{*}{$\begin{array}{l}\text { Mentha spp., incl. Mentha } \\
\text { xpiperita L., Lamiaceae } \\
\text { (LUUB011) }\end{array}$} & \multirow[t]{7}{*}{ м'ята / mjata } & \multirow{7}{*}{$\begin{array}{l}\text { aerial } \\
\text { parts }\end{array}$} & decoction & sedative & 5 \\
\hline & & & tea & cough & 2 \\
\hline & & & & headache & 1 \\
\hline & & & & heart problems & 2 \\
\hline & & & & hemorroids & 1 \\
\hline & & & & sedative & 1 \\
\hline & & & topical applicaton & wounds & 1 \\
\hline \multirow{5}{*}{$\begin{array}{l}\text { Nepeta cataria L., } \\
\text { Lamiaceae (LJUB072) }\end{array}$} & \multirow[t]{5}{*}{ меліса / mielisa } & \multirow{5}{*}{$\begin{array}{l}\text { aerial } \\
\text { parts }\end{array}$} & \multirow[t]{5}{*}{ tea } & sedative & 1 \\
\hline & & & & cancer & 1 \\
\hline & & & & kidney diseases & 1 \\
\hline & & & & panacea & 1 \\
\hline & & & & stomachache & 1 \\
\hline \multirow[t]{3}{*}{$\begin{array}{l}\text { Salvia officinalis L., } \\
\text { Lamiaceae (LJUB025) }\end{array}$} & \multirow[t]{3}{*}{ шалфей / šalfiej } & $\begin{array}{l}\text { aerial } \\
\text { parts }\end{array}$ & tea & panacea & 1 \\
\hline & & leaves & tea & toothache & 1 \\
\hline & & & & diarrhea & 1 \\
\hline \multirow{2}{*}{$\begin{array}{l}\text { Vicia faba } L ., \\
\text { Leguminosae }\end{array}$} & \multirow[t]{2}{*}{ боб, фасоль / bob, fasol } & \multirow[t]{2}{*}{ beans } & \multirow[t]{2}{*}{ boiled } & diarrhea & 1 \\
\hline & & & & diarrhea in cows (vet) & 1 \\
\hline \multirow{7}{*}{$\begin{array}{l}\text { Linum usitatissimum } \mathrm{L} ., \\
\text { Linaceae }\end{array}$} & лён / lion & seeds & boiled & diarrhea in cows (vet) & 7 \\
\hline & & & & diarrhea in pigs (vet) & 5 \\
\hline & & & decoction & $\begin{array}{l}\text { rumination problems in } \\
\text { cows (vet) }\end{array}$ & 8 \\
\hline & & & & good for pigs (vet) & 2 \\
\hline & & & dried & $\begin{array}{l}\text { constipation in cows } \\
\text { (vet) }\end{array}$ & 1 \\
\hline & & & oil & men's diseases & 1 \\
\hline & & & tea & stomachache & 2 \\
\hline Althaea officinalis L., Malvaceae & алтэй / altej & aerial & tea & cold & 1 \\
\hline & & parts & & sedative & 1 \\
\hline
\end{tabular}


Table 1 Medicinal and ethnoveterinary uses of cultivated plants (Continued)

\begin{tabular}{|c|c|c|c|c|c|}
\hline $\begin{array}{l}\text { Plant taxon, family } \\
\text { (voucher specimen code) }\end{array}$ & Local names & $\begin{array}{l}\text { Used } \\
\operatorname{part}(s)\end{array}$ & Preparation & $\begin{array}{l}\text { Recorded medical } \\
\text { use(s) (treated disease) }\end{array}$ & UC \\
\hline & & & fed fresh & $\begin{array}{l}\text { rumination problems in } \\
\text { cows (vet) }\end{array}$ & 2 \\
\hline Paeonia spp., Paeoniaceae & $\begin{array}{l}\text { піон укланяюшчійся / pion } \\
\text { uklaniajuščijsia }\end{array}$ & roots & $\begin{array}{l}\text { alcohol } \\
\text { maceration }\end{array}$ & sedative & 1 \\
\hline Cedrusspp., Pinaceae & кедр / kiedr & nuts & dried & $\begin{array}{l}\text { macerated in strong } \\
\text { alcohol }\end{array}$ & 1 \\
\hline \multirow[t]{4}{*}{ Piper nigrum L., Piperaceae } & \multirow[t]{4}{*}{$\begin{array}{l}\text { перац горкі гарошкам, перац чорны / pierac } \\
\text { horki haroškam, pierac čorny }\end{array}$} & \multirow[t]{4}{*}{ seeds } & fed & $\begin{array}{l}\text { weakness in chickens } \\
\text { (vet) }\end{array}$ & 1 \\
\hline & & & fresh & diarrhea & 2 \\
\hline & & & \multirow{2}{*}{$\begin{array}{l}\text { mixed with } \\
\text { alcohol, drunk }\end{array}$} & cold & 2 \\
\hline & & & & rhinitis & 1 \\
\hline \multirow[t]{2}{*}{ Avena sativa L., Poaceae } & \multirow[t]{2}{*}{ авёс / avios } & shoots & decoction & liver diseases & 2 \\
\hline & & grains & decoction & cold & 1 \\
\hline Oryza sativa L., Poaceae & pic / ris & grains & decoction & diarrhea & 3 \\
\hline Zea mays L., Poaceae (LUUD018) & кукуруза / kukuruza & silk & tea & liver diseases & 1 \\
\hline \multirow{12}{*}{$\begin{array}{l}\text { Aronia spp. (Aronia } \times \text { prunifolia (Marshall) } \\
\text { Rehder (LUUB033) and Aronia melanocarpa } \\
\text { (Michx.) Elliott), Rosaceae }\end{array}$} & \multirow{12}{*}{$\begin{array}{l}\text { аронія, рабіна чорная, рабіна чарнаплодная, } \\
\text { чарнаплодка / aronija, rabina čornaja, rabina } \\
\text { čarnaplodnaja, čarnaplodka }\end{array}$} & \multirow{11}{*}{ fruits } & boiled & diarrhea in pigs (vet) & 1 \\
\hline & & & \multirow[t]{2}{*}{ dried } & diarrhea & 2 \\
\hline & & & & panacea & 1 \\
\hline & & & \multirow[t]{3}{*}{ fresh } & hypertension & 2 \\
\hline & & & & hyperthyroidism & 2 \\
\hline & & & & diarrhea & 1 \\
\hline & & & \multirow{2}{*}{$\begin{array}{l}\text { alcohol } \\
\text { maceration }\end{array}$} & hypertension & 2 \\
\hline & & & & wellbeing & 1 \\
\hline & & & \multirow[t]{3}{*}{ tea } & diarrhea & 2 \\
\hline & & & & hypertension & 1 \\
\hline & & & & liver diseases & 1 \\
\hline & & leaves & tea & hypertension & 1 \\
\hline $\begin{array}{l}\text { Chaenomeles japonica (Thunb.) Lindl. ex } \\
\text { Spach, Rosaceae }\end{array}$ & айва японская / ajva japonskaja & fruits & jam, raw & wellbeing & 1 \\
\hline \multirow[t]{3}{*}{ Prunus cerasus L., Rosaceae (LJUB097) } & \multirow[t]{3}{*}{ вішня, вішняк / višnia, višniak } & leaves & tea & sore throat & 1 \\
\hline & & \multirow[t]{2}{*}{ twigs } & \multirow[t]{2}{*}{ tea } & cold & 2 \\
\hline & & & & $\begin{array}{l}\text { strenghtening of } \\
\text { organism }\end{array}$ & 1 \\
\hline Pyrus communis L., Rosaceae & груша / hruša & fruits & dried & diarrhea & 4 \\
\hline Citrus limon (L.) Osbeck; Rutaceae & лімон / limon & fruits & fresh & cold & 1 \\
\hline $\begin{array}{l}\text { Capsicum annuum L., Solanaceae } \\
\text { (LJUD015) }\end{array}$ & красный перец / krasnyj pieriec & fruits & $\begin{array}{l}\text { alcohol } \\
\text { maceration, } \\
\text { topical application }\end{array}$ & joint pain & 1 \\
\hline Nicotiana spp., Solanaceae & табак / tabak & leaves & tea, grugling & toothache & 1 \\
\hline \multirow[t]{6}{*}{ Solanum tuberosum L., Solonaceae } & \multirow{6}{*}{$\begin{array}{l}\text { бульба, картофель, картошка / buíba, } \\
\text { kartofiel, kartoška }\end{array}$} & flowers & topical applicaton & joint pain & 1 \\
\hline & & \multirow[t]{5}{*}{ tubers } & boiled & diarrhea in calves (vet) & 1 \\
\hline & & & candles & hemorroids & 3 \\
\hline & & & compress & eye inflamation & 2 \\
\hline & & & \multirow[t]{2}{*}{ fresh, fodder } & $\begin{array}{l}\text { for cows, produce } \\
\text { more milk }\end{array}$ & 2 \\
\hline & & & & fodder for pigs & 1 \\
\hline
\end{tabular}


Table 1 Medicinal and ethnoveterinary uses of cultivated plants (Continued)

\begin{tabular}{|c|c|c|c|c|c|}
\hline $\begin{array}{l}\text { Plant taxon, family } \\
\text { (voucher specimen code) }\end{array}$ & Local names & $\begin{array}{l}\text { Used } \\
\text { part(s) }\end{array}$ & Preparation & $\begin{array}{l}\text { Recorded medical } \\
\text { use(s) (treated disease) }\end{array}$ & UC \\
\hline & & & $\begin{array}{l}\text { smashed hot, } \\
\text { applied on chest }\end{array}$ & cough & 1 \\
\hline & & & inhalation of & rhinitis & 5 \\
\hline & & & bollıng water & cold & 2 \\
\hline & & & & cough & 1 \\
\hline & & & topical applicaton & burns & 3 \\
\hline & & & of tresh slices & skin diseases & 2 \\
\hline \multirow[t]{4}{*}{ Camellia sinensis (L.) Kuntze, Theaceae } & \multirow{4}{*}{$\begin{array}{l}\text { чай (крепкі), чай чорны пакупны / } \\
\text { čaj (kriepki), čaj čorny pakupny }\end{array}$} & \multirow{4}{*}{$\begin{array}{l}\text { leaves } \\
\text { from } \\
\text { shop }\end{array}$} & compress & eye problems & 7 \\
\hline & & & decoction & $\begin{array}{l}\text { eye inflamation in } \\
\text { cats (vet) }\end{array}$ & 1 \\
\hline & & & eaten dried & diarrhea & 1 \\
\hline & & & tea & diarrhea & 1 \\
\hline Vitis spp., Vitaceae & вінаград / vinahrad & fruits & fresh & hypotension & 1 \\
\hline \multirow{14}{*}{$\begin{array}{l}\text { Aloe spp. (mainly Aloe arborescens Mill.), } \\
\text { Xanthorrhoeaceae }\end{array}$} & \multirow{14}{*}{$\begin{array}{l}\text { альвас, гальяс, алоэ, сталетнік / } \\
\text { alvas, haljas, aloe, stalietnik }\end{array}$} & \multirow[t]{6}{*}{ leaves } & \multirow{2}{*}{$\begin{array}{l}\text { eaten fresh, } \\
\text { mixed with honey }\end{array}$} & cold & 3 \\
\hline & & & & stomachache & 1 \\
\hline & & & \multirow{2}{*}{$\begin{array}{l}\text { alcohol } \\
\text { maceration }\end{array}$} & lung diseases & 3 \\
\hline & & & & tuberculosis & 1 \\
\hline & & & \multirow[t]{2}{*}{ topical applicaton } & wounds & 3 \\
\hline & & & & burns & 2 \\
\hline & & \multirow[t]{8}{*}{ sap } & cooked & lung diseases & 1 \\
\hline & & & drunk fresh & cold & 1 \\
\hline & & & \multirow[t]{5}{*}{ topical applicaton } & inflamation & 1 \\
\hline & & & & cuts & 1 \\
\hline & & & & joint pain & 1 \\
\hline & & & & rotten wounds & 1 \\
\hline & & & & wounds & 4 \\
\hline & & & washed with & beauty procedure & 1 \\
\hline
\end{tabular}

The gastrointestinal category, while having a relatively low FIC value ( 0.59 for 25 taxa), contained seven taxa that met the reliability criterion: Anethum graveolens (to alleviate flatulence and stomach ache), Aronia spp. (to relieve stomach ache), Beta vulgaris (to cure constipation), Pyrus communis and Oryza sativa (to treat diarrhea), Piper nigrum (to treat diarrhea and stomach ache), Solanum tuberosum (to treat hemorrhoids). Within the other disease categories only a few individual taxa met the reliability criterion, among these: Allium sativum in the oral and dental category (to treat toothache); Mentha spp. (as a sedative and to relieve headache) and Brassica oleracea (against headache) in the neurological category; Brassica oleracea (to alleviate joint pain) in the musculoskeletal category; and $A l$ lium sativum and Ribes nigrum in the general health category.

The majority of the temporary, past and adulthood uses of cultivated plants overlap with uses that have been continuously practiced; however, some differences do exist. For example, some plants were reported to be used differently in the past than they are now, including the tea of cultivated Mentha species which in the past was drunk to treat hemorrhoids, but now is used in adulthood by many people as a sedative (also used continuously) and to treat respiratory illnesses. The use of Mentha leaves applied on wounds was also only practiced in the past. However, uses of cultivated plant taxa practiced only in the past were rather rare and included the freshly pressed oil of Cannabis sativa which was drunk to cure constipation and the tea of Nicotiana spp. which was gurgled to alleviate toothache. A few additional taxa were mentioned only when talking about uses acquired during adulthood; for example, Capsicum annuum macerated in alcohol to treat rheumatic diseases or raw jam of Chaenomeles japonica to promote wellbeing, roots of Helianthus tuberosus eaten raw to treat diabetes, and tea of the leaves of Hyssopus 
officinalis to combat cold and stomach problems - all of which are known from the widespread popular literature on home-medication and widely promoted in the media (for example, the most popular newspaper "Narodnyy Doktor" publishes readers" recipes that are sent to the editorial office).

One temporary use also stood out, although the plant itself was continuously used: children were bathed in a decoction of twigs and leaves of Ribes nigrum against diathesis. The person describing the use mentioned that the wild plant (three-lobe beggarticks, Bidens tripartita L.) did not help her children, "but the leaves of blackcurrant did".

Out of the 134 people interviewed, 73 mentioned using cultivated or store-bought plants for medication. Twenty-five people mentioned only one use, while the most knowledgeable person mentioned 16 taxa and three more individuals recalled 13 taxa. The mean number of used taxa was 3.6, which is relatively quite low, especially considering that almost all households had a vegetable garden, indicating that plants were cultivated.

Comparison with the available historical data While the historical data on the use of cultivated plants for healing is relatively limited, some comparisons can be drawn. For example, the use of intensely tasting plants have retained their popularity, even if the specific use indicated has changed considerably in nature: at the end of nineteenth century it was believed that onion and garlic would frighten off diseases, as well as protect children and pregnant women, and also newlyweds during their wedding $[40,43,44,62]$, whereas now only a few interviewees recalled the use of garlic (as a necklace) as a means of maintaining good health and protecting against cold, while the primary emphasis of the modern use of fresh onion and garlic is on respiratory diseases. In the nineteenth century garlic was used to relieve teething pain in infants $[43,62]$, while now it is used to treat toothache. The specific use of baked onions has also changed as now it is used mainly to treat dermatological problems, while according to nineteenth century sources mothers used to rub the new-born's breast with baked onions to alleviate rhinitis [63].

\section{Non-plant remedies}

There were 188 DUR referring to the use of 58 different remedies (Table 2). The six most popular remedies included vodka (19 DUR), pork fat and honey (both 14 DUR), salt (11 DUR), propolis (10 DUR), and goat milk (9 DUR); however, only vodka was named by more than $10 \%$ of interviewees.

Altogether 93 UI were recorded in general disease categories. The reliability criterion (at least three users) was met by 21 general UI. This group contained 16 different remedies, of which only five were used in two disease categories. The most popular was vodka, used in the respiratory and gastrointestinal categories, followed by goat milk in the general health and respiratory categories, propolis in the general health and respiratory categories, salt in the respiratory and nephrological and urological categories, and goose fat in the respiratory and dermatological categories. Among the remaining 11 remedies, only three were reported by at least five people: pork fat used to treat oral and dental diseases, honey in the case of respiratory diseases and oil in the gastrointestinal category.

The domestic use of non-plant remedies on the individual level was rather restricted, as only one person claimed using spirits in four different illness categories, while two more individuals used honey for treating three different ilnesses.

The informant consensus factor for the whole medicinal use area of other remedies was very low $(\mathrm{FIC}=0.67$, 175 use citations for 58 remedies). All but three general disease categories had lower FIC values.

The cardiovascular category contained only one remedy: dead honeybees $(\mathrm{FIC}=1)$ (Figs. 3, 4). The oral and dental disease category had the next highest FIC value $(0.85)$ and contained one dominant remedy (pork fat, either fresh or salted) and two less frequently used ones (topical applications of salt and vodka on an aching tooth). Another small general disease category with a FIC slightly lower than that for the whole use area (0.66) was nephrological and urological ailments, containing only two remedies (brandy drunk to treat kidney stones and salted hot water applied externally on cystitis). The respiratory disease category was the only extensive one that had a relatively high FIC value (0.68). Among a relatively wide variety of remedies (16), seven met the reliability criterion (having more than three users). Of these the most popular was honey, used mainly to treat cough but also sore throat, and vodka, which was drunk to relieve cold, cough and rhinitis, as well as applied topically to alleviate earache. Goat milk and cow milk with honey and/or soda were used mainly to relieve cough (in children), and heated salt was applied topically to treat sinusitis, sore throat, earache and cough (socks with hot salt). Also, goose fat was mixed with honey and applied or smeared on the chest to alleviate cough and rhinitis.

The gastrointestinal disease category $(\mathrm{FIC}=0.52,14$ remedies) contained only four remedies that met the reliability criterion. The most popular of these was the use of oil to relieve constipation, vodka to treat stomach ache and diarrhea, kefir to allieviate constipation and starch to cure diarrhea. The general health category, containing seven remedies (FIC $=0.53$ ), encompassed only two remedies that met the reliability criterion: the use of goat milk to treat allergies in children as well as 
Table 2 Non-plant remedies used to treat humans and other animals

\begin{tabular}{|c|c|c|c|c|}
\hline Remedy & Local names & Preparation & Application & $U$ \\
\hline Anthill & куча мурав'ёф / kuс̌a muravjof & ritual, literature based, bath & epilepsy & 1 \\
\hline \multirow[t]{2}{*}{ Beer } & \multirow[t]{2}{*}{ nisa / piva } & forced to drink & rumination problems in cow (vet) & 3 \\
\hline & & poured on stones in sauna & aromatherapy & 1 \\
\hline Bile of the pig & жәлчь am свіней / želć at sviniej & $\begin{array}{l}\text { macerated in alcohol, topical } \\
\text { application }\end{array}$ & pain & 1 \\
\hline Brandy & каньяк / kańjak & drunk & kidney stones & 1 \\
\hline \multirow[t]{2}{*}{ Bread } & \multirow[t]{2}{*}{ хлеб / chlieb } & \multirow[t]{2}{*}{ mumbled on, allowed to eat } & $\begin{array}{l}\text { to protect cows against snake bites } \\
\text { (vet) }\end{array}$ & 1 \\
\hline & & & to protect cows (vet) & 1 \\
\hline $\begin{array}{l}\text { Bread, soft part of } \\
\text { it }\end{array}$ & мякіш хлеба / miakiš chlieba & $\begin{array}{l}\text { roll over the stomach, say words and } \\
\text { then fed to a dog }\end{array}$ & fright & 1 \\
\hline Bread mold & ивілы хлеб / ćvily chlieb & $\begin{array}{l}\text { scratched from bread, mixed with fat, } \\
\text { smeared }\end{array}$ & mastitis in cows (vet) & 1 \\
\hline Brick & кірпічына / kirpičyna & $\begin{array}{l}\text { sit on a bucket with hot water and hot } \\
\text { brick }\end{array}$ & women's diseases & 1 \\
\hline Broken hay, chaff & дзёран / dzioran & fodder & vitamins for calves (vet) & 2 \\
\hline Butter & масла / masla & heated until boiling, eaten & diarrhea & 1 \\
\hline Chicken navel skin & $\begin{array}{l}\text { плёначкі ад курыных пупкоў / plionački } \\
\text { ad kurynych pupkoŭ }\end{array}$ & dried, macerated in hot water & diarrhea & 1 \\
\hline \multirow[t]{3}{*}{ Clay } & \multirow[t]{3}{*}{ гліна / hlina } & fresh, fodder & given to calves (vet) & 2 \\
\hline & & diluted with water, forced to drink & $\begin{array}{l}\text { when an overheated horse has drunk } \\
\text { cold water (vet) }\end{array}$ & 1 \\
\hline & & fresh, additional fodder & pigs, to make joints strong (vet) & 1 \\
\hline $\begin{array}{l}\text { Cow milk, freshly } \\
\text { milked }\end{array}$ & сырадой / syradoj & washed with & eye inflammation & 1 \\
\hline \multirow[t]{2}{*}{ Diesel oil } & \multirow{2}{*}{$\begin{array}{l}\text { дзізельнае масла, аўтол / dzizielnaje } \\
\text { masla, aŭtol }\end{array}$} & forced to drink & rumination problems in cow (vet) & 1 \\
\hline & & topical application & skin diseases in pigs and piglets (vet) & 1 \\
\hline Dog hair & шэрсuь сабакі / šersć sabaki & cut and set on fire & fright & 2 \\
\hline \multirow{3}{*}{$\begin{array}{l}\text { Dung water or } \\
\text { horse feces }\end{array}$} & \multirow{3}{*}{$\begin{array}{l}\text { конскі гной, конскі кал / konski hnој, } \\
\text { konski kal }\end{array}$} & dropped on sugar, eaten & helminthic infection & 1 \\
\hline & & fed & helminthic infection in pigs (vet) & 3 \\
\hline & & $\begin{array}{l}\text { few drops put into glass of water, so } \\
\text { person drinking it does not know }\end{array}$ & helminthic infection & 3 \\
\hline \multirow[t]{3}{*}{ Egg } & \multirow[t]{3}{*}{ яйка / jajka } & boiled, applied hot & allergic rhinitis & 1 \\
\hline & & boiled, applied hot & sinusitis & 1 \\
\hline & & boiled & diarrhea (in children) & 2 \\
\hline Egg and vodka & яйчо з гарэлкаю / jajco z harelkaju & mixed and forced to drink & diarrhea in piglets (vet) & 3 \\
\hline Egg yolk & яічный жэлток / jaičnyj želtok & $\begin{array}{l}\text { salve made with oil of Helianthus } \\
\text { annuus and egg yolk }\end{array}$ & panacea & 1 \\
\hline Egg white & бялок яйца / bialok jajca & applied fresh & burns & 1 \\
\hline Fat of the badger & барсучья сала / barsućja sala & melted and smeared & burns & 1 \\
\hline Fat of the goose & гусіны жыр / husiny žyr & $\begin{array}{l}\text { mixed with honey and applied with } \\
\text { cabbage leaves on chest }\end{array}$ & cough in children & 3 \\
\hline \multirow[t]{5}{*}{ Fat of the pig } & \multirow[t]{5}{*}{ сала / sala } & fresh, topical application & foot sores & 1 \\
\hline & & fresh, topical application & toothache & 5 \\
\hline & & salted, topical application & toothache & 6 \\
\hline & & smeared on scalp & to increase hair growth & 1 \\
\hline & & topical application & mastitis in cows (vet) & \\
\hline
\end{tabular}


Table 2 Non-plant remedies used to treat humans and other animals (Continued)

\begin{tabular}{|c|c|c|c|c|}
\hline Remedy & Local names & Preparation & Application & $U C$ \\
\hline & & $\begin{array}{l}\text { ointment made with turpentine, } \\
\text { topical application }\end{array}$ & aching legs & 1 \\
\hline Fat of the ram & барані жыр / barani žyr & $\begin{array}{l}\text { mixed with hot milk or honey } \\
\text { and vodka }\end{array}$ & cough & 1 \\
\hline \multirow[t]{2}{*}{ Fat of the sheep } & авечы жыр / aviečy žyr & added to hot milk & cold in children & 1 \\
\hline & & $\begin{array}{l}\text { salve made with root of Arctium } \\
\text { spp. }\end{array}$ & joint pain & 1 \\
\hline \multirow[t]{4}{*}{ Fat of the goose } & гусіны жыр / husiny žyr & smeared & burns & 2 \\
\hline & & & cold & 1 \\
\hline & & & earache & 1 \\
\hline & & & foot sores & 1 \\
\hline $\begin{array}{l}\text { Fat of the } \\
\text { hedgehog }\end{array}$ & вожыка жыр / vožyka žyr & boiled, smeared & burns & 1 \\
\hline Fireplace grime & сажа з коміна / saža z komina & forced to eat & diarrhea in calves (vet) & 1 \\
\hline Flour & мука / muka & fodder additive & $\begin{array}{l}\text { to increase milk production in cows } \\
\text { (vet) }\end{array}$ & 3 \\
\hline Grasshopper & $\begin{array}{l}\text { конік, кузнечык, ивыркун / } \\
\text { konik, kuźniečyk, cvyrkun }\end{array}$ & topical application & warts & 2 \\
\hline \multirow[t]{10}{*}{ Honey } & мёд / miod & $\begin{array}{l}\text { diluted with water, topical } \\
\text { application }\end{array}$ & eye problems & 1 \\
\hline & & $\begin{array}{l}\text { mixed with goose fat and applied } \\
\text { with cabbage leaves on chest }\end{array}$ & cough in children & 3 \\
\hline & & eaten & cold & 2 \\
\hline & & & cough & 1 \\
\hline & & & panacea & 1 \\
\hline & & & sore throat & 1 \\
\hline & & & stomach ache & 1 \\
\hline & & topical application & burns & 1 \\
\hline & & mixed with hot milk, drunk & cough & 1 \\
\hline & & mixed with vodka, applied on throat & sore throat & 1 \\
\hline Honeybees & пчала / pčala & allowed to sting & rheumatism & 1 \\
\hline \multirow[t]{3}{*}{ Honeybees, dead } & пчаліны падмур / & macerated in alcohol (for 2 weeks) & heart problems & 2 \\
\hline & pcalliny padmur & & hypertension & 2 \\
\hline & & & joint pain & 1 \\
\hline Kefir & kecbip / kiefir & drunk & constipation & 3 \\
\hline Lactic acid & малочная кіслата / maločnaja kislata & forced to drink & rumination problems in cows (vet) & 1 \\
\hline \multirow[t]{5}{*}{ Laundry soap } & хазяйсивеннае мыла / chaziajscviennaje & ground, compress, external & sore throat & 2 \\
\hline & & ground, clyster & constipation in cows (vet) & 3 \\
\hline & & smeared & mastitis in cows (vet) & 1 \\
\hline & & topical application & wounds & 1 \\
\hline & & smeared & mastitis in cows (vet) & 5 \\
\hline $\begin{array}{l}\text { Leftovers from } \\
\text { moonshine } \\
\text { distillation }\end{array}$ & бpaza / braha & forced to drink & stomach ache in cows (vet) & 1 \\
\hline $\begin{array}{l}\text { Liquid left after } \\
\text { making curds }\end{array}$ & $\begin{array}{l}\text { сыроватка (сываратка) / syrovatka } \\
\text { (syvaratka) }\end{array}$ & forced to drink & rumination problems in cows (vet) & 1 \\
\hline Lice & Bom / voš & hidden in bread and given to eat & jaundice & 1 \\
\hline
\end{tabular}


Table 2 Non-plant remedies used to treat humans and other animals (Continued)

\begin{tabular}{|c|c|c|c|c|}
\hline Remedy & Local names & Preparation & Application & UC \\
\hline $\begin{array}{l}\text { Meat of the } \\
\text { badger }\end{array}$ & барсук / barsuk & boiled and eaten & tuberculosis & 1 \\
\hline \multirow[t]{3}{*}{ Meat of the dog } & сабака / sabaka & dried, eaten & tuberculosis & 1 \\
\hline & & made into cutlets & tuberculosis & 1 \\
\hline & & boiled and eaten & tuberculosis & 1 \\
\hline \multirow[t]{3}{*}{ Milk of the cow } & малако / malako & garlic added and sit in hot bath & helminthic infection & 1 \\
\hline & & forced to drink & stomach problems in cows (vet) & 1 \\
\hline & & heated, soda added, drunk & sore throat & 1 \\
\hline $\begin{array}{l}\text { Milk of the cow, } \\
\text { sour }\end{array}$ & кіслае малако / kislaje malako & drunk & constipation & 2 \\
\hline \multirow[t]{8}{*}{ Milk of the goat } & казінае малако / kazinaje malako & drunk & allergies in children & 3 \\
\hline & & drunk hot & cough in children & 3 \\
\hline & & topical application & eye pain & 1 \\
\hline & & drunk & cancer & 1 \\
\hline & & drunk & asthma & 1 \\
\hline & & heated, drunk with honey & earache & 1 \\
\hline & & & cough & 3 \\
\hline & & heated, drunk with soda & cough & 3 \\
\hline Motor oil & маторнае масла / matornaje masla & forced to drink & stomach problems in cows (vet) & 1 \\
\hline $\begin{array}{l}\text { Mussel shells or } \\
\text { chalk }\end{array}$ & ракушкі иі мел / rakuški ci miel & broken, fodder additive & to make egg-shells stronger (vet) & 1 \\
\hline \multirow[t]{2}{*}{ Oil } & алей / aliej & drunk & constipation & 5 \\
\hline & & forced to drink & rumination problems in cows (vet) & 2 \\
\hline Petroleum & керасін, кірасін / kierasin, kirasin & compress & sore throat & 2 \\
\hline \multirow[t]{2}{*}{ Petrol } & бензін, салярка / bienzin, saliarka & applied on scratched warts & warts & 1 \\
\hline & & topical application & eczema & 1 \\
\hline \multirow{2}{*}{$\begin{array}{l}\text { Potassium } \\
\text { permanganate }\end{array}$} & марганиоўка / marhancoŭka & drunk & vomiting & 1 \\
\hline & & weak solution, drunk & diarrhea & 1 \\
\hline \multirow[t]{4}{*}{ Propolis } & праполіс / prapolis & $\begin{array}{l}\text { macerated in alcohol, topical } \\
\text { application }\end{array}$ & joint pain & 4 \\
\hline & & macerated in alcohol, drunk & panacea & 2 \\
\hline & & & stomach ache & 1 \\
\hline & & macerated in strong alcohol & sore throat & 1 \\
\hline Rain water & дажджавая вада / daždžavaja vada & washed with & hair care & 1 \\
\hline \multirow[t]{9}{*}{ Salt } & соль / sol & dissolved in water, gurgling & toothache & 1 \\
\hline & & heated, topical application & cystitis & 3 \\
\hline & & & earache & 1 \\
\hline & & & toothache & 1 \\
\hline & & & sore throat & 1 \\
\hline & & heated and put into socks & cough & 1 \\
\hline & & mixed with vodka, drunk & diarrhea & 1 \\
\hline & & $\begin{array}{l}\text { mixed with rye flour, heated, topical } \\
\text { application }\end{array}$ & sinusitis & 1 \\
\hline & & ritual "salting" & evil eye & 1 \\
\hline Salted water & салёная вада / salionaja vada & side of the calve pierced and the & rumination problems in calves (vet) & 1 \\
\hline
\end{tabular}


Table 2 Non-plant remedies used to treat humans and other animals (Continued)

\begin{tabular}{|c|c|c|c|c|}
\hline Remedy & Local names & Preparation & Application & $U$ \\
\hline Snakeskin & змяіная кожа / zmiainaja koža & dried, topical application & burns & 1 \\
\hline Snakeskin (old) & $\begin{array}{l}\text { змеіная лінь (старая шкура) / zmieinaja } \\
\text { liń (staraja škura) }\end{array}$ & topical application & pimples & 1 \\
\hline $\begin{array}{l}\text { Sand where } \\
\text { chickens bathe }\end{array}$ & $\begin{array}{l}\text { пясок, дзе куры купаючиа / } \\
\text { piasok, dzie kury kupajuсcа }\end{array}$ & topical application & warts & 1 \\
\hline $\begin{array}{l}\text { Sebaceous } \\
\text { secretion of goose }\end{array}$ & гусіны гной / husiny hnој & $\begin{array}{l}\text { smeared on with leaves, topical } \\
\text { application }\end{array}$ & splinter in the finger & 1 \\
\hline Snailshells or chalk & ракушкі ці мел / rakuški ci miel & broken, fodder additive & $\begin{array}{l}\text { to make chicken egg-shells stronger } \\
\text { (vet) }\end{array}$ & 1 \\
\hline \multirow[t]{6}{*}{ Soda } & \multirow[t]{6}{*}{ coda / soda } & bath & foot sores & 1 \\
\hline & & gurgling with solution & sore throat & 2 \\
\hline & & mixed with water, drunk & cleaning of stomach & 1 \\
\hline & & mixed with water, eyes washed & eye pain & 1 \\
\hline & & mixed with water, forced to drink & rumination problems in goats (vet) & 1 \\
\hline & & topical application & burns & 1 \\
\hline \multirow[t]{7}{*}{ Spirits } & \multirow[t]{7}{*}{ cnipm / spirt } & drink one teaspoon every day & $\begin{array}{l}\text { against a fright from lightening, not to } \\
\text { be nervous and nasty throughout life }\end{array}$ & 1 \\
\hline & & smeared & sore throat & 1 \\
\hline & & topical application & cold & 1 \\
\hline & & & eczema & 1 \\
\hline & & & joint pain & 1 \\
\hline & & & psoriasis & 1 \\
\hline & & & sore throat & 1 \\
\hline \multirow[t]{3}{*}{ Starch } & \multirow[t]{3}{*}{ крахмал / krachmal } & $\begin{array}{l}\text { eaten dry and water drunk } \\
\text { afterwards }\end{array}$ & diarrhea & 1 \\
\hline & & mixed with cold water, drunk & diarrhea & 2 \\
\hline & & $\begin{array}{l}\text { mixed with cold water, forced to } \\
\text { drink }\end{array}$ & diarrhea in calves (vet) & 1 \\
\hline \multirow[t]{2}{*}{ Sugar } & \multirow[t]{2}{*}{ caxap / sachar } & \multirow[t]{2}{*}{ topical application } & eye pain & 1 \\
\hline & & & wounds & 1 \\
\hline Sweat of horses & коHCKi nom / konski pot & topical application & warts & 1 \\
\hline \multirow[t]{2}{*}{ Tar } & \multirow[t]{2}{*}{ дзёгаць, maвот / dziohać, tavot } & \multirow[t]{2}{*}{ smeared } & red fever in pigs (vet) & 1 \\
\hline & & & scabs in pigs (vet) & 1 \\
\hline Trichlorfon & хларафос / chlarafos & diluted, topical application & skin diseases in pigs & 1 \\
\hline Turpentine & unikidap / špikidar & $\begin{array}{l}\text { topical application on navel; } \\
\text { dripped on sugar, eaten }\end{array}$ & helminthic infection & 1 \\
\hline \multirow[t]{7}{*}{ Urine } & \multirow[t]{7}{*}{ мача / mača } & compress & joint pain & 1 \\
\hline & & drunk & fright & 1 \\
\hline & & smeared & mastitis in cows (vet) & 4 \\
\hline & & \multirow[t]{4}{*}{ topical application } & cancer & 1 \\
\hline & & & cuts & 1 \\
\hline & & & wounds & 1 \\
\hline & & & wounds in horses (vet) & 1 \\
\hline Water, cold & халодная вада / chalodnaja vada & topical application & headache & 1 \\
\hline Wet cloth & мокрая трапка / mokraja trapka & put on cow stomach & rumination problems in cows (vet) & 1 \\
\hline \multirow[t]{2}{*}{ Window "sweat" } & \multirow[t]{2}{*}{ nот на вакне / pot na vaknie } & \multirow[t]{2}{*}{ topical application } & eczema & 1 \\
\hline & & & burns & \\
\hline
\end{tabular}


Table 2 Non-plant remedies used to treat humans and other animals (Continued)

\begin{tabular}{|c|c|c|c|c|}
\hline Remedy & Local names & Preparation & Application & UC \\
\hline Vinegar & уксус / uksus & topical application & warts & 3 \\
\hline \multirow{20}{*}{$\begin{array}{l}\text { Vodka / } \\
\text { moonshine }\end{array}$} & \multirow{20}{*}{$\begin{array}{l}\text { гарэлка, водка, самагонка / } \\
\text { harelka, vodka, samahonka }\end{array}$} & applied on hole & toothache & 1 \\
\hline & & \multirow[t]{3}{*}{ compress } & back pain & 1 \\
\hline & & & foot ache & 1 \\
\hline & & & sore throat & 1 \\
\hline & & \multirow[t]{3}{*}{ drunk } & diarrhea & 1 \\
\hline & & & panacea & 1 \\
\hline & & & stomach ache & 1 \\
\hline & & \multirow[t]{6}{*}{ forced to drink } & diarrhea in pigs (vet) & 1 \\
\hline & & & dog plague (vet) & 2 \\
\hline & & & goat kids' illness (vet) & 1 \\
\hline & & & helminthic infection in dogs (vet) & 1 \\
\hline & & & rumination problems in cows (vet) & 4 \\
\hline & & & stomach ache in cows (vet) & 2 \\
\hline & & \multirow[t]{3}{*}{ smeared } & fever & 1 \\
\hline & & & mastitis in cows (vet) & 2 \\
\hline & & & stomach ache & 1 \\
\hline & & smeared on soles & cough & 1 \\
\hline & & gurgled & toothache & 1 \\
\hline & & salted a bit and drunk & diarrhea & 1 \\
\hline & & topical application & earache & 2 \\
\hline \multirow[t]{4}{*}{ Vodka and pepper } & \multirow{4}{*}{$\begin{array}{l}\text { гарелка з периам / } \\
\text { harielka z piercam }\end{array}$} & \multirow[t]{4}{*}{ mixed, drunk } & cold & 2 \\
\hline & & & rhinitis & 1 \\
\hline & & & diarrhea & 1 \\
\hline & & & stomach ache & 1 \\
\hline $\begin{array}{l}\text { Vodka, diluted with } \\
\text { human urine }\end{array}$ & $\begin{array}{l}\text { водка разбавленая челавеческай мачёй / } \\
\text { vodka razbavlienaja čielaviečieskaj mačioj }\end{array}$ & given to drink & $\begin{array}{l}\text { when a person drinks too much } \\
\text { alcohol }\end{array}$ & 1 \\
\hline Yeast & дрожжы / drožžy & forced to eat & stomach ache in cows (vet) & 1 \\
\hline
\end{tabular}

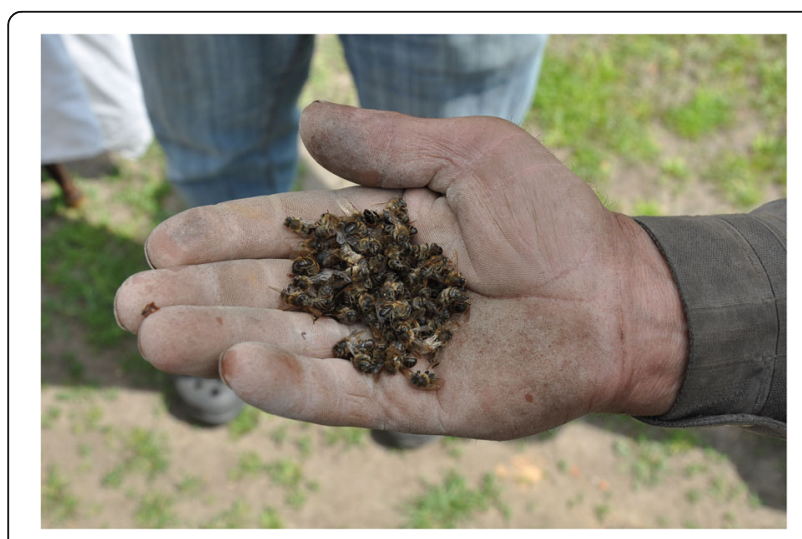

Fig. 3 Dead honeybees. Plastok village, photo Raivo Kalle. 13.05 .2016 to combat cancer, and propolis macerated in alcohol as a panacea. The infection disease category ( $\mathrm{FIC}=0.5$, five remedies) contained only one remedy that met the reliability criterion: horse dung water used to treat helminthic infection.

Within the remaining general disease categories FIC values were very low $(0-0.3)$, with only use in the dermatology category meeting the reliability criterion: goose fat smeared on burns and foot sores.

A few temporary uses were related to curing particular personal diseases encountered only once in life or during a specific period, such as treating sick children or having access to a remedy (like using fresh milk to treat eye inflammation while keeping a personal cow). Quite a number of recently acquired uses seem to have a traditional origin (e.g. the use of old snakeskins to treat pimples or rolling the soft part of the bread over the stomach, saying a few words and then giving the bread to dogs to alleviate fright), but they 


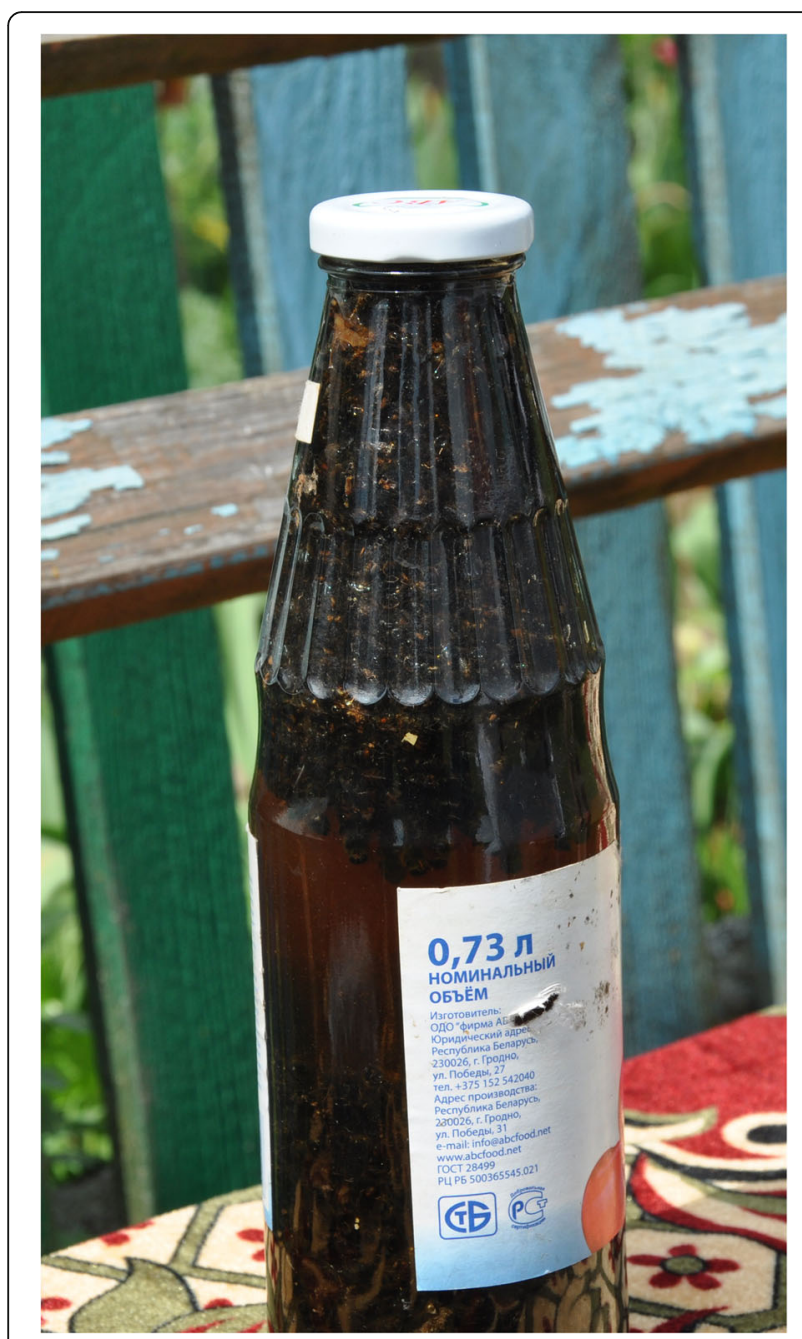

Fig. 4 Dead honeybees macerated in moonshine. Plastok village, photo Raivo Kalle. 13.05.2016

could also have been learned from books or journals promoting "traditions", such as the myth of treating epilepsy with anthills [13]. The popularity of honey and propolis as a medicine is now supported by popular literature (7 uses out of 10 for propolis and 7 out of 8 for honey were attributed to recent acquisition) and may also be due to the massive promotion of apicultural products as good for health [6466]. Many past uses are spatially distributed among the most popular uses, except a few: burning dog hair to alleviate fright; sheep fat to treat cold and in a salve to alleviate joint pain; lice hidden in bread and given to eat to treat jaundice; hot milk with garlic as a bath to cure helminthic infection; rainwater for washing hair; sweat of horses or sand where chickens bathe to treat warts - all of these, however, were named by only one or two people.

Out of the 134 individuals participating in the study, 60 mentioned the use of other remedies for human medication and of these 23 mentioned only one remedy.
The two most knowledgeable individuals recalled 19 and 10 DUR and 17 and 6 used remedies, respectively. The mean number of DUR was 3.3 and for remedies it was 2.7 .

\section{Comparison with the available historical data}

The historical legacy concerning non-plant remedies is much richer than that of cultivated plants which indicates that this remedy group has clearly decreased in number and also in use nomenclature. Historically, symbolic practices of the treatment of diseases were associated with the magical properties of water, fire and stones (soil), and water treatment was widespread [63, 67]. In Belarusian ethnomedicine water was considered a universal remedy in the treatment of almost all diseases, on the basis of both its basic potential to purify the body and the repetitive healing through sanctification, etc. [37]. As common within all traditional medicines around the world, water had, in the opinion of Belarusians, specific powers to heal through bathing (flushing, pouring out). Belarusians treated with water "rosette" (acute pains in the stomach) [43], "evil eye" [43, 63, 68], and a variety of skin diseases [62, 63]; however, such uses could also have been influenced (or earlier local use supported) by widespread hydrotherapy ideas propagated by popular literature, for example the book on water treatment by German doctor Sebastian Kneip (1821-1897). Moreover, the addition of salt [43], metal products [43, 62], coins [43], and ash [69], among other things, was believed to add extra magical powers to water. The first time a child was bathed a coin was thrown into the water, which according to magical beliefs would provide health, skin purity and wealth to the child. One interviewee did recall such a treatment when asked specifically, but her reference was quite impersonal, "second hand" and therefore it is not referred to in the analyzed data, although in other regions of Belarus the use of different metals has been recorded (iron, silver [37], etc.).

Historically fire was an important factor in the process of the symbolic "overbaking" of a sick child in a furnace, a ritual common during the nineteenth century in Hrodna, Minsk and Vicebsk provinces [43, 45, 70]. Likewise, fumigation or smoke was used to cure a fever [39, 69]. Moreover, the historical widespread re-use of ritual objects for fumigation (for example, candles [41, 43, 62] and shells of Easter eggs [62]) was not mentioned by the participants in the current study.

The topical application of stones, widespread historically $[45,62,63,71]$, seems to be largely forgotten, as is the use of all naturally occuring substances apart from salt; for example, minerals (silica [62]), silver (coins [41, $45,67])$ and clay $([41,45])$. The only exceptions noted in this study were the recorded use of sand in which chickens bathed for treating warts, which was clearly 
indicated as a recalled past use, and the ingestion of clay associated with ethnoveterinary use.

Vodka, however, used historically to dilute some other substances [20,39], has become a very popular remedy on its own and as a dissolving agent in combination with a variety of plants and, on occasion, non-plant remedies (tinctures for internal or external use).

The use of plant-based products unidentified on the species level, such as the earlier widespread use of $\operatorname{tar}$ [41], was only occasionally mentioned. Another plant-based product, bread, was historically attributed protective properties and used in the treatment of various diseases [41, $45,63,72]$; however, this study recorded the use of bread for treatment only a few times, and in all cases it was used as a mediation substance, conveying words (mumbled on and fed to cows) or as a symbolic means of hiding healing substances (e.g. the remembered past use of hiding lice to treat jaundice). Interestingly, Vallejo and González [73] showed that the use of bread with lice in Spain was encountered in the literature only between 1942 and 1972, although the researched literature extended from 1927 to 2012. The use of lice for the treatment of jaundice was widespread among Belarusians [37]. One recent use (reported as practiced during adulthood, but not during childhood) was the use of bread as a medium for the transfer of fright from humans to dogs. The so-called "out-rolling" with the soft part of bread while still hot was a historically well known magical remedy among Belarusians and other Slavic peoples and used predominantly to remove "fright" from the body. The bread was rolled over the back or stomach and as a result it collected a lot of fur and hair - often used to diagnose the agent "responsible" for the disease. These "out-rolled" diseases were then carried outside human habitats (thrown into water, taken to the forest, left on crossroads) or, more often, fed to dogs. Other substances (such as ashes, wool or boiled eggs) were also sometimes used for the same purpose [74, 75].

Products of animal origin as well as their metabolic products were occasionally used or recalled as having been used in childhood; and all of these were used historically as well. The most common among these included fat [42, 43, 62], apiculture products [63], dairy products [62], and eggs [39, 41, 43, 45, 46, 62, 63, 67, 76], as well as urine [41] and dung water of horse feces and bile [42, 43]. Yet the historical repertorie was much more diverse, containing many products not recalled during our interviews, such as horns and blood [63], feathers [63, 70], placenta [63], waste matter [43, 62, 63], wool and bones [43] and some of the internal organs [63].

The list of the historically used domestic animals and birds is very long, and includes horse [63, 68], pig [45, 63], cow [63], chicken [68], goat [42], cat [63], bull [42], a variety of small mammals (rat [63], bats [43], hare [42], hedgehog [63]), wild birds (cuckoo [42]), reptiles (snake
[43, 68], lizard [43]), amphibians (frog [42, 43, 63]), insects (bees [38, 63, 69]) and also fishes (burbot [42], pike $[43,77])$. Although it is not always clear how exactly the animal was used in the past, the use of animals often relied on a basic principle - treating similar with similar, with the aim to "transmit" diseases to animals, both by means of direct contact as well as through charms and magical actions (fumigation of wool or feathers of an animal/bird that caused the consternation $[63,69]$ ). Among the records of the present study, mainly practical and seemingly quite rational uses of animal (products) have continued to be used.

Among the human-originated substances, the historical data list such remedies as human placenta [67], hair $[38,41,63]$, nails $[41,45,63]$, and the urine of sick people [20,43]. Items bearing symbolism of the underworld include, for example, objects belonging to the deceased or a cemetery $[43,45,62]$, spider's web $[43,45]$, moss (from the roof of the house) $[43,77]$ and a piece of cloth with menstrual blood [77]. None of these were mentioned during the interviews in this study.

\section{Charm-healing in the context of lay-treatment}

The recording of charms was not the purpose of the present study as healing with charms and healing with plants are usually perceived as separate categories of healing, considered independent. Yet in a few cases we encountered some elements of healing concerned with the use of charms that are related to the use of nonplant remedies, such as alleviating fright by rolling bread over the stomach. The use of plants both in magical practices and as accompainments to charms is based not only on their real biological characteristics, but also on the laws of mythological logic - the place of the plant in the traditional worldview [78]. The use of plants in folk medicine is often based on the principle of signatura rerum, when people believe that the plant itself (by its color, shape, etc.) indicates which disease they need to treat. In magical practice in general, the correlation of the external manifestations of a disease, especially the cutaneous ones, with a particular plant is important. This is particularly evident in the example of the ritual removal of warts, for which comparably round potatoes, peas and apples were used: "People took potatoes and rubbed them on the warts. Potatoes cut in half, applied on the warts, and then pressed together and buried. As soon as the potato dries the warts should disappear" [37]. All across the country this practice continues to use prickly, scalding or even poisonous plants as apotropes, for fumigating a patient with the magical intention of expelling the spirit of the disease. For example, children that had received a fright were fumigated with agrimony, among other things. Other features of the plant have become more relevant, such as the 
multiplicity of poppy and flax seeds, when sprinkled over a child afflicted by the evil eye, etc.

Treatment with words, which was embodied in the practice of medical charms, was historically considered an important way to get rid of disease along with remedies of plant, animal and mineral origin. Charms were mainly used as prophylactics and to treat children's and women's diseases, infectious diseases, skin diseases, neuropsychiatric conditions, dental issues and complaints related to the digestive system, head, eyes, ears, nose and throat, as well as fear, the evil eye, and health problems related to childbirth. This type of treatment was conducted by healers, although some proportion of the population had basic folk medical knowledge. The charms are accompanied by certain actions which reinforce the magical meaning of the act of treating.

Earlier folklore collectors noticed that "treatments with charms exist everywhere and belief in them was unusually high" [20]. During fieldwork for this study many interviewees mentioned as a past phenomenon whispering old women (бабка-шаптуха, babka-šaptucha) who were consulted in the course of specific diseases (such as fright and evil eye). However, a few lay interviewees also recited some charms. One of the villages even had their own healer man, who was still practicing healing with charms. Today the practice of applying to healers is reserved for illnesses of the psycho-neurological sphere (fright, evil eye, insomnia) and certain skin diseases (for example, erysipelas).

\section{Veterinary medication \\ Cultivated plants}

There were 48 DUR referring to the use of 14 taxa from 12 families, of which only two families, Asteraceae and Cucurbitaceae, were represented by two taxa. The most commonly used taxa, and the only one named by 14 people, was Linum usitatissimum (18 DUR). Three additional taxa met the reliability criterion: Solanum tuberosum, Cucumis sativus and Anethum graveolens (three users and DUR for each). The diversity of uses on the individual level was very low: one person used two plants and five people used one plant for treating two different animal illnesses.

The informant consensus factor for the whole veterinary use area of cultivated plants was relatively low, yet similar to the FIC of wild plants (FIC $=0.69$, 43 use citations for 14 taxa). The only individual general category with the same FIC value was the emic category rumination problems, for which six taxa were used; however, only one of them, Linum usitatissimum, met the reliability criterion (eight users). All other disease categories had low FIC values within the range of $0.2-0.56$. The only two uses that met the reliability criterion within those categories consisted of Linum usitatissimum to treat diarrhea in both cows (seven users) and pigs (five users).

There were no extraordinary uses of cultivated plants that have been attributed only to past or present contexts, except for the recent adoption of the feeding seeds of Piper nigrum to chickens in order to strengthen them. The taxa Linum usitatisiiumum was temporally dynamic: two uses were claimed to have been acquired during adulthood (rumination problems and diarrhea in cows) and seven uses were attributed to the past, of which six were divided equally among diarrhea in cows, diarrhea in pigs and rumination problems in cows; however, the same uses were also continuously utililized.

Out of the 134 individuals participating in the study, 26 mentioned the use of cultivated plants for veterinary purposes. The most knowledgeable person mentioned four taxa (and six DUR), whereas 18 people mentioned only one taxon. The mean number of used cultivated taxa was 1.4 while the mean DUR for this group was 1.7.

Comparison with the available historical data Most popular or widespread cultivated plants noted in nineteenth century literature appear to be no longer used. Some of them (such as Nicotiana spp. which was indicated for the treatment of cow, horse and sheep diseases [48, 49, 52]; and Cannabis sativa [48-50] which was used to treat dogs, horses and sheep) have been officially banned for domesti cultivation in different periods of 20th century, whereas others (such as some cereals (Secale cereale, Hordeum vulgare, Avena sativa, Fagopyrum esculentum) [48] used for cow, horse and pig healing) simply stopped being grown in home-gardens due to fundamental changes in the economic sphere occurring in the twentieth century. Other historically known cultivated plants include garlic which was used for the treatment of wounds (especially on the tongue of ruminants) as well as against sheep parasites (матыліиь) $[48,51,52]$ and various vegetables (e.g. radish [49], horseradish, cabbage, carrot [48]) used to treat internal diseases of cows and horses. None of these was used by our interviewees.

\section{Non-plant remedies}

In ethnoveterinary medicine, 72 DUR referring to the use of 31 non-plant remedies were identified. The most commonly used remedies were vodka (13 DUR) and laundry soap (eight DUR). Twenty remedies were used by one person only. Five additonal remedies met the reliability criterion: urine (five users), egg and vodka, beer, horse feces and clay (three users each). The diversity of uses was almost as low as for cultivated plants: one person used vodka for three different emic animal illnesses, while six more people used various remedies for treating two different emic animal illnesses. 
The informant consensus factor for the whole veterinary use area of non-plant remedies was higher than for the human medication use area of non-plant remedies and similar to the FIC of cultivated and wild plants ( $\mathrm{FIC}=0.57,70$ use citations for 31 remedies). Only two of the individual general categories had FIC values higher than that for the whole use area.

Mastitis was the disease category with the highest FIC value (0.71); and in this category five different remedies were used, two of which, laundry soap and urine, met the reliability criterion. The category of helminthic infection had a FIC value of 0.66 and contained two remedies, of which the use of horse feces met the reliability criterion. All other disease categories had low FIC values within the range $0.18-0.5$. Within these only a few uses met the reliability criterion: vodka and beer were forcefed to cows to treat rumination problems, a mixture of eggs and vodka was given to piglets to cure diarrhea and laundry soap was given to cows to alleviate constipation.

Over $40 \%$ of the DUR were described as used in the past; the majority of them were among the most widely used remedies, while some were uniquely used remedies, such as pouring salted water into the perforated side of a calf or giving soda to goats to drink to treat rumination problems, and the smearing of pigs with tar to cure red fever or scabs. Over $60 \%$ of the uses of vodka were also attributed to the past (when animals were still kept by many people). None of the uses in this remedy category were reported as recently acquired.

Out of the 134 individuals interviewed, 30 mentioned the use of non-plant remedies for veterinary purposes, and of these people 12 mentioned only one remedy. The most knowledgeable person mentioned eight remedies, and two other individuals mentioned five remedies and DUR. The mean number of non-plant remedies was 2.3 while the mean DUR of this group was 2.4.

Comparison with the available historical data Significant quantitative predominance of non-plant remedies over cultivated plants used for treating domestic animals seems also to be historically specific for Belarusian ethnoveterinary medicine. Some of these remedies were still in use or recalled as past uses. The universal non-plant remedies (stones $[48,52]$, water $[48,49,53]$, fire $[48,50$, $54]$, bread $[48,50,52]$, salt $[48,50]$ etc.), which have already been discussed above in the context of ethnomedicine, were also widely used in veterinary medicine for both treating and preventing animal diseases (mastitis, rabies in dogs, snakebites, digestive disorders of cows and horses, cow placental retention (retentio placentae), skin diseases of cattle and horses, "the evil eye", etc.). The most numerous and diverse group of nonplant remedies consisted of animal-based ones, such as different kinds of animals and parts of their bodies (insects [48, 49, 52, 53, 55], birds [49], mammals [48, 49, $52,53]$, fishes [48, 52], amphibians [48, 48]] and reptiles [48]) or substances obtained from human and animal bodies (fat $[49,52]$, urine $[48,50,52]$, feces $[48,49,51$, $52]$, placenta $[49,52]]$, bile $[48,49]$, blood $[49,52])$ that were used to treat ungulates (horses, cows, sheep and pigs). Honey [48-51, 54] and the application of birch tar $[48,49,56]$ played an important role in treating infectious diseases of horses, cows and pigs. Various milk products were used to treat ungulate skin diseases [49, $51]$, as well as urination [49] and digestive [48, 50] problems in cattle. Eggs were considered to be a universal remedy for cow and horse diseases $[49,52]$.

Comparison between the use of wild and cultivated plants and non-plant remedies While modern complimentary and alternative medicine has been perceived as a self-driven choice and possibility for caring for one's self [79], the "remains" of traditional healing practices have a kind of ambivalent position, valued as traditional relicts, yet often perceived as something less effective, and even shameful (for example, the use of urine, and even the use of vodka). As many (especially wild) plants used in ethnomedicine belonged to the official repertoire of Soviet medicine, it seems that this group of home remedies had been silently given "use consent" in home-healing.

All human disease categories have been predominantly treated with wild plants and only two categories, namely culture bound and oral and dental, had more extensive use of non-plant remedies, although the nomenclature of the remedies for dental treatment was rather limited (Fig. 5, Table 3).

In ethnoveterinary medicine, non-plant remedies have been relatively more important than both wild and cultivated plant remedy groups. However, only one ethnoveterinary category, namely mastitis, has been treated solely with non-plant remedies, while another (rumination problems) boasts both the largest number of uses and the greatest diversity within the non-plant remedies (Table 4, Fig. 6). Cultivated plants have had modest application in ethnoveterinary medicine, slightly more prominent in the diversity of taxa used only in the category of gastrointestinal problems other than rumination.

The entire diversity in the use of both ethnomedicinal and ethnoveterinary remedies was created by only a limited number of people, providing many use records (Fig. 7). Even though there were a remarkable number of people (41) who named only wild plants used in human ethnomedicine, there were also six people who named only cultivated plants and/or non-plant remedies. The situation for ethnoveterinary medicine differs slightly as a similar proportion of individuals used only non-plant 

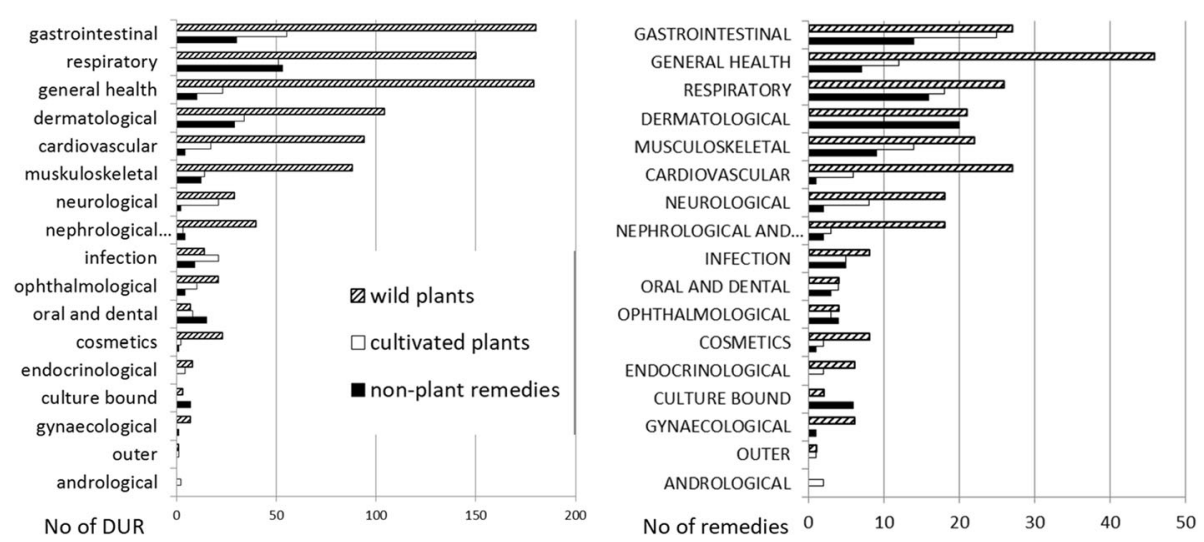

Fig. 5 Division of the number of DURs and used remedies within three remedy groups and different human disease categories; on both sides of the graph disease categories are listed according to the sum of all DURs or used remedies in the category

remedies (eight people), cultivated plants (seven people) or both (six people), and a relatively smaller proportion (compared to human medicine) of people (14 individuals) used only wild plants to attend animals. The occurrence of people in the sample who have used only nonplant remedies indicates that, even if asking about wild food plants first may have predisposed some respondents to concentrate on wild plants, it was not universal.

In human ethnomedicine wild plants constitute the most changeable group of remedies, with over $40 \%$ of uses adopted later in the life (Fig. 8). Other remedies contribute the highest proportion of continuous uses as well as the highest proportion of the past uses, while recently acquired uses represent the lowest proportion. Cultivated plants fall in between the other two remedy groups, contributing a very small proportion of past uses, while recent additions seem to be quite numerous, but still representing a smaller proportion than among wild plants. Among the remedy groups in the ethnoveterinary category the difference regarding the recently acquired uses is noteworthy: almost $30 \%$ of the uses of wild plants in contrast to none for non-plant remedies. The non-plant remedy group also boasts the greatest proportion (over $40 \%$ ) of uses attributed to the past only. None of the uses in the ethnoeterinary use area were referred to as temporary.

Comparison of the overlap of remedies in the human medicinal and ethnoveterinary use areas shows an interesting anomaly in the non-plant remedies category. A vast majority (all but three taxa) of the wild plants and all but one cultivated plant used in ethnoveterinary medicine were also used for human medication. Among non-plant remedies the overlap is considerably less: 14 remedies out of 31 used for ethnoveterinary medicine did not have any other use in human medicine.

Therefore, non-plant remedies can be perceived as the remnants of traditional healing which is no longer evolving. As this group is not supported by official medicine, it has less new uses (acquired during adulthood) and more past uses, and the group may continue to diminish, as has already happened compared to the widespread historical use in Belarus. The more extensive use of non-plant remedies in the ethnoveterinary use area also supports this idea, as ethnoveterinary medicine in Belarus seems to be of marginal importance due to welldeveloped and accessible professional veterinary care and thus it is practiced by few enthusiasts only.

What supports sustainability of wild plants in human medicine and non-plant remedies in ethnoveterinary medicine? Wild plants are more versatile because of their availability, diversity and sustainability with regard to the local climate. A significant number of uses of wild plants are associated with knowledge obtained from the media (such as Narodnaya gazeta [59], 7 dnej [60]) as well as from the older generation. For example, publications have promoted the use of twigs of trees and berry bushes for tea [61], berries for compresses, rinses and baths [62], and inflorescences for decoctions and ointments [63].

About 33\% of the study area is covered by forests, $27.5 \%$ of which are man-made, mainly consisting of coniferous plantations. Coniferous, birch, and black-alder forests, as well as oak and fir trees can be encountered. In the mid-1920s, a large land reclamation initiative began in the territory of the district. The peak of melioration fell on 1960-1970s [64]. In total, more than 70,000 ha of swamps were drained in the Liuban region alone. As a result of land reclamation, the area of agricultural land increased significantly (the total area of agricultural land is now 79.2 thousand hectares, of which 62.3 thousand ha are drained); however, due to a number of mistakes and miscalculations, some parts of this land began to be covered with sand dunes, on which 
Table 3 Distribution of emic disease categories among general disease categories and different domains. UCcp - Use Citation of cultivated platns, UCnonp -Use Citation of nonplant remedies, UCwp -Use Citation of wild plants. UUT/R - sum of used plant taxa / other remedies; FIC - Informant Consensus Factor

Emic disease categories / General disease UCcp UCnonp UCwp
category - EUT/R (FIC)

\begin{tabular}{|c|c|c|c|}
\hline Andrological & $2(0)$ & 0 & 0 \\
\hline Men's diseases & 1 & & \\
\hline Potency & 1 & & \\
\hline Cardiovascular & $6(0.64)$ & $1(1)$ & $27(0.62)$ \\
\hline Anemia & & & 1 \\
\hline Bad blood & & & 1 \\
\hline Haemostatic & & & 4 \\
\hline Heart diseases & & & 9 \\
\hline Heart problems & 9 & 2 & 34 \\
\hline Hypertension & 7 & 2 & 34 \\
\hline Hypotension & 1 & & 3 \\
\hline Promotes bloodstream & & & 2 \\
\hline Thickening of blood & & & 1 \\
\hline Varix & & & 4 \\
\hline Vesical cleaning & & & 1 \\
\hline Cosmetics & $2(0)$ & $1(0)$ & $8(0.67)$ \\
\hline Rejuvenating & & & 2 \\
\hline Beauty procedure & 1 & & 1 \\
\hline Hair care & 1 & 1 & 19 \\
\hline Dandruff & & & 1 \\
\hline Culture bound & 0 & $6(0.17)$ & $2(0.5)$ \\
\hline Against a fright from lightening & & 1 & \\
\hline Evil eye & & 1 & 1 \\
\hline Fright & & 5 & 1 \\
\hline For women to be strong against men & & & 1 \\
\hline Dermatological & $10(0.69)$ & $20(0.3)$ & $21(0.81)$ \\
\hline Abscesses & 3 & & 4 \\
\hline Allergies & & & 1 \\
\hline Burns & 11 & 9 & 7 \\
\hline Cuts & 2 & 1 & 7 \\
\hline Eczema & & 2 & 2 \\
\hline Foot sores & & 3 & 3 \\
\hline Inflammation & 1 & & 1 \\
\hline Inflammation after injection & 1 & & \\
\hline Pimples & & 1 & \\
\hline Psoriasis & & 1 & \\
\hline Rotten wounds & 2 & & 1 \\
\hline Scabies & & & 1 \\
\hline
\end{tabular}

Table 3 Distribution of emic disease categories among general disease categories and different domains. UCcp - Use Citation of cultivated platns, UCnonp -Use Citation of nonplant remedies, UCwp -Use Citation of wild plants. $\Sigma U T / R$ - sum of used plant taxa / other remedies; FIC - Informant Consensus Factor (Continued)

Emic disease categories / General disease UCcp UCnonp UCwp category - $\Sigma$ UTT/R (FIC)

\begin{tabular}{llll}
\hline Skin diseases & 4 & & 4 \\
Splinter in the finger & & 1 & \\
Tumor & & & 1 \\
Warts & & 8 & 10 \\
Wounds & 10 & 3 & 62 \\
Endocrinological & $2(0.5)$ & 0 & $6(0.29)$ \\
Diabetes & 2 & & 5 \\
Hyperthyroidism & 2 & & 2 \\
Pancreas & & & 1 \\
Gastrointestinal & $25(0.59)$ & 14 & $27(0.78)$ \\
& & $(0.52)$ &
\end{tabular}

Bile neutralizer

Bile deficiency

Cleaning of stomach

Constipation

$\begin{array}{lll}7 & 10 \quad 4\end{array}$

Gastritis

Gastric ulcer

Gall stones

Dysentery

Diarrhea

$20 \quad 12 \quad 63$

Flatulence (in children)

Hemorrhoids

Jaundice

Laxative

Liver diseases

Low acidity

Stomach problems

Stomach ache

Vomiting

General health

Allergies

Appetizer

Aroma therapy

Body cleansing

2

Bone strengthening

Cancer

CO-intoxication

Diathesis in children

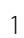

1

3

17

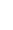

1

$12(0.5) \quad 7(0.54) \quad 46(0.75)$

2

3

1

5

1

3

2

12 
Table 3 Distribution of emic disease categories among general disease categories and different domains. UCcp - Use Citation of cultivated platns, UCnonp -Use Citation of nonplant remedies, UCwp -Use Citation of wild plants. $\mathrm{UTT} / \mathrm{R}$ - sum of used plant taxa / other remedies; FIC - Informant Consensus Factor (Continued)

Emic disease categories / General disease UCcp UCnonp UCwp category - ¿UT/R (FIC)

\begin{tabular}{|c|c|c|c|}
\hline Disinfectant & & & 3 \\
\hline Fever & & 1 & 3 \\
\hline Good for health & & & 6 \\
\hline Hangover & & & 1 \\
\hline Wellbeing & 7 & & 49 \\
\hline Immune boosting & 4 & & 7 \\
\hline Inflammation processes & & & 5 \\
\hline Men's health & 1 & & \\
\hline Organism cleansing & & & 7 \\
\hline Pain & & & 1 \\
\hline Panacea & 5 & 7 & 32 \\
\hline Prophylactics & 1 & & 26 \\
\hline Strengthening of organism & 2 & & 4 \\
\hline Tonus support & & & 1 \\
\hline Washing hair & & & 1 \\
\hline Vitamins & & & 4 \\
\hline Gynecological & 0 & $1(0)$ & $6(0.17)$ \\
\hline Women's diseases & & 1 & 6 \\
\hline To increase human milk production & & & 1 \\
\hline Infection & $5(0.79)$ & $5(0.5)$ & $8(0.46)$ \\
\hline Anti-microbic & & & 1 \\
\hline Chickenpox & & & 1 \\
\hline Helminthic infection & 20 & 6 & 9 \\
\hline Scabies & & & 1 \\
\hline Tuberculosis & 1 & 3 & 2 \\
\hline Musculoskeletal & $14(0.54)$ & $9(0.2)$ & $22(0.74)$ \\
\hline Back pain & & 1 & 7 \\
\hline Bruises & & & 3 \\
\hline Foot ache & & 1 & 8 \\
\hline For adhesion of bones & & & 1 \\
\hline Joint pain & 12 & 9 & 50 \\
\hline Knee ache & & & 2 \\
\hline Rheumatic pains & 2 & 1 & 17 \\
\hline Nephrological and urological & $3(0)$ & $2(0.67)$ & $18(0.54)$ \\
\hline Cystitis & & 3 & \\
\hline Diuretic & 1 & & 7 \\
\hline Kidney diseases & 1 & & 29 \\
\hline Kidney stones & 1 & 1 & 1 \\
\hline Urinary bladder problems & & & 2 \\
\hline
\end{tabular}

Table 3 Distribution of emic disease categories among general disease categories and different domains. UCcp - Use Citation of cultivated platns, UCnonp -Use Citation of nonplant remedies, UCwp -Use Citation of wild plants. $\Sigma U T / R$ - sum of used plant taxa / other remedies; FIC - Informant Consensus Factor (Continued)

Emic disease categories / General disease UCcp UCnonp UCwp category - $\Sigma$ UTT/R (FIC)

\begin{tabular}{|c|c|c|c|}
\hline Urinating problems & & & 1 \\
\hline Neurological & $8(0.59)$ & $2(0)$ & $18(0.35)$ \\
\hline Calming & & & 6 \\
\hline Convulses & & & 1 \\
\hline Epilepsy & & 1 & 4 \\
\hline Headache & 8 & 1 & 11 \\
\hline Insomnia & & & 1 \\
\hline Nerves & & & 2 \\
\hline Sedative & 12 & & 1 \\
\hline Soporific & 1 & & 3 \\
\hline Ophthalmological & $3(0.78)$ & $4(0)$ & $4(0.84)$ \\
\hline Eye inflammation & 2 & 1 & \\
\hline Eye pain & 2 & 2 & \\
\hline Eye problems & 6 & 1 & 16 \\
\hline Improve vision & & & 5 \\
\hline Oral and dental & $4(0.57)$ & $3(0.85)$ & $4(0.5)$ \\
\hline Gingival inflammation & 1 & & \\
\hline Gingival bleeding & & & 2 \\
\hline Gingival diseases & & & 1 \\
\hline Gingival wound & & & 1 \\
\hline Periodontitis & & & 1 \\
\hline Teething pain in children & & & 1 \\
\hline Toothache & 7 & 15 & 1 \\
\hline Other & $1(0)$ & 0 & $1(1)$ \\
\hline Antitoxic & & & 1 \\
\hline Snake bites & 1 & & \\
\hline Respiratory & $18(0.63)$ & $\begin{array}{l}16 \\
(0.68)\end{array}$ & $26(0.84)$ \\
\hline Asthma & & 1 & \\
\hline Bronchitis & & & 6 \\
\hline Cold & 19 & 7 & 94 \\
\hline Cough & 8 & 23 & 42 \\
\hline Earache & 3 & 5 & 1 \\
\hline Expectorant & & & 1 \\
\hline Lung diseases & 4 & & 2 \\
\hline Pneumonia & & & 2 \\
\hline Rhinitis & 13 & 2 & 2 \\
\hline Sinusitis & & 2 & \\
\hline Sore throat & 4 & 13 & \\
\hline
\end{tabular}


Table 4 Distribution of emic ethnoveterinary categories among general ethnoveterinary categories and different means of treatment. UCcp - Use Citation of cultivated plants, UCnonp Use Citation of non-plant remedies, UCwp -Use Citation of wild plants. UUT/R - sum of used plant taxa / other remedies; FIC Informant Consensus Factor

\begin{tabular}{|c|c|c|c|}
\hline $\begin{array}{l}\text { Emic etnoveterinary categories / General } \\
\text { ethnoveterinary category - } \Sigma U T / R(F I C)\end{array}$ & UCcp & UCothr & UCwp \\
\hline Gastrointestinal & $9(0.56)$ & $5(0.5)$ & $8(0.63)$ \\
\hline Constipation in cows & 1 & 3 & \\
\hline Diarrhea in calves & 1 & 2 & 2 \\
\hline Diarrhea in chickens & & & 1 \\
\hline Diarrhea in cows & 9 & & 10 \\
\hline Diarrhea in piglets & & 3 & \\
\hline Diarrhea in pigs & 7 & 1 & 8 \\
\hline Fodder & $5(0.2)$ & $3(0.33)$ & $10(0.55)$ \\
\hline Fodder for bees in spring & & & 1 \\
\hline Fodder for cows & & & 3 \\
\hline Fodder for home animals & & & 2 \\
\hline Fodder for pigs & 2 & & 5 \\
\hline Fodder for rabbits & & & 4 \\
\hline Fodder for turkeys & & & 3 \\
\hline $\begin{array}{l}\text { Fodder to increase milk production in } \\
\text { cows }\end{array}$ & 4 & 3 & 1 \\
\hline Fodder to make egg-shells stronger & & 2 & \\
\hline Helminthic infection & 0 & $2(0.67)$ & $1(0)$ \\
\hline Helminthic infection in pigs & & 3 & 1 \\
\hline Helminthic infection in dogs & & 1 & \\
\hline Helminthic infection in cows & & & 1 \\
\hline Mastitis & 0 & $5(0.71)$ & 0 \\
\hline Mastitis in cows & & 15 & \\
\hline Rumination problems & $6(0.69)$ & $13(0.4)$ & $3(0.67)$ \\
\hline Rumination problems in calves & & 1 & \\
\hline Rumination problems in cow & 17 & 11 & 6 \\
\hline Rumination problems in goats & & 1 & 1 \\
\hline Stomach ache in cows & & 4 & \\
\hline Stomach problems in cows & & 4 & \\
\hline Strengthening of animals & $1(0)$ & $3(0.33)$ & $4(0.5)$ \\
\hline Given to claves & & 2 & \\
\hline Goat kids' illness & & 1 & \\
\hline Strengthening of cows & & & 1 \\
\hline Strengthening of piglets & & & 1 \\
\hline Strengthening of pigs & & 1 & 4 \\
\hline Vitamins for cows & & & 1 \\
\hline Vitamins for claves & & 2 & \\
\hline Weakness in chickens & 1 & & \\
\hline
\end{tabular}

Weakness in chickens
Table 4 Distribution of emic ethnoveterinary categories among general ethnoveterinary categories and different means of treatment. UCcp - Use Citation of cultivated plants, UCnonp Use Citation of non-plant remedies, UCwp -Use Citation of wild plants. $\Sigma U T / R$ - sum of used plant taxa / other remedies; FIC Informant Consensus Factor (Continued)

\begin{tabular}{|c|c|c|c|}
\hline $\begin{array}{l}\text { Emic etnoveterinary categories / General } \\
\text { ethnoveterinary category - } \Sigma U T / R(F I C)\end{array}$ & UCcp & UCothr & UCwp \\
\hline Other & $5(0.43)$ & $10(0.18)$ & $8(0.3)$ \\
\hline Appetizer for domestic animals & 1 & & 1 \\
\hline Appetizer for cows & & & 2 \\
\hline Bacterial diseases in bees & & & 1 \\
\hline Blood in urine in cows & & & 1 \\
\hline Cold in domestic animals & & & 1 \\
\hline Cuts in domestic animals & & & 1 \\
\hline Disinfection for home animals & & & 1 \\
\hline Dog plague & & 2 & \\
\hline Eczema & & 1 & \\
\hline Eye inflammation in cats & 1 & & \\
\hline Eye pain & & 1 & \\
\hline Good for cows & & & 1 \\
\hline Good for domestic animals & 1 & & \\
\hline Good for horses & & & 1 \\
\hline Good for pigs & 4 & & \\
\hline Red fever in pigs & & 1 & \\
\hline Scabs & & 1 & \\
\hline Skin diseases in pigs and piglets & & 1 & \\
\hline Skin diseases in pigs & & 1 & \\
\hline To protect cows & & 1 & \\
\hline To protect cows against snake bites & & 1 & \\
\hline $\begin{array}{l}\text { When an overheated horse has drunk } \\
\text { cold water }\end{array}$ & & 1 & \\
\hline $\begin{array}{l}\text { When piglets do not go to their } \\
\text { mother }\end{array}$ & & & 1 \\
\hline Wounds in cows & & & 1 \\
\hline Wounds in horses & & 1 & \\
\hline
\end{tabular}

nothing grew. Today, marshes cover $0.7 \%$ of the Liuban region [14].

In the territory of the Liuban district there are 3 hospitals, a polyclinic, 10 dispensaries, 14 first aid points, and 6 pharmacies. As a rule, pharmacies are located in towns. In villages, medicines can only be purchased at first aid stations [14]. Veterinary help is provided by veterinarians who work in collective farms, as well as specialists who are retired [80]. In spite of the wellorganized professional assistance available, folk veterinary medicine, or a set of methods based on both natural science knowledge about animals and the traditional mythopoetic model of the world, continues to actively 

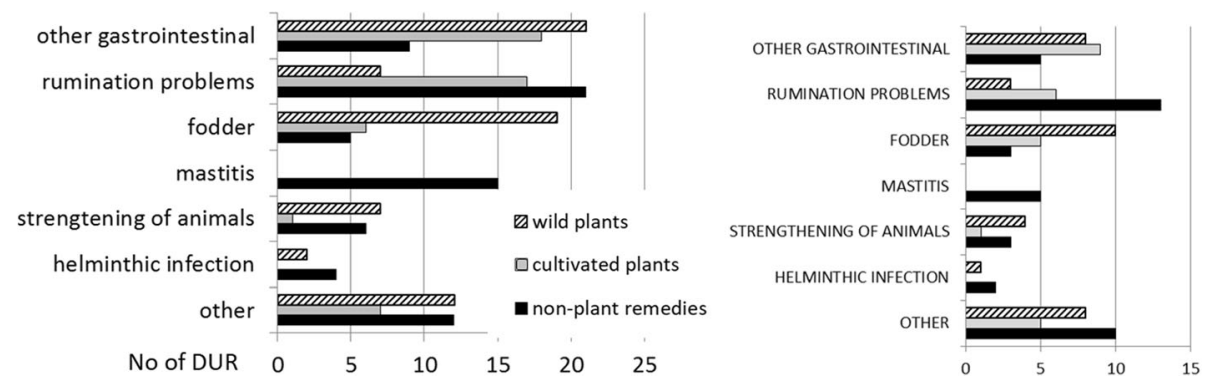

Fig. 6 Division of the numbers of DURs and used remedies within three remedy groups and different ethnoveterinary categories; on both sides of the graph disease categories are listed according to the sum of all DURs or used remedies in the category

adhere to folk remedies, or the use of vegetable, animal, mineralogical, and magical medicinal products, including charms. The latter decreased, in comparison with the post-war period, by the 1980s (the time of active collection of material in the Paliessie region). This was due to urbanization and the decline of rural households as well as ideological changes in society, which brought about the decay of traditional culture. At the same time a vast store of traditional knowledge about animal treatment is preserved in the passive memory of rural residents. Also, their use may be encouraged by veterinarians, a significant portion of who are of rural origin themselves and thus they use "grandmother's" remedies in their professional activities. Non-plant remedies may also still be important in veterinary practice due to the influence of popular literature (magazines, newspapers) and media.

Knowledge and skills from the field of folk medicine, apart from the use of plant and animal derived products, tend to be in the realm of the sacred. In regard to Soviet atheism they were painted in a negative light with the label "superstition" and pushed underground. This may also be the reason why the respondants in this study did not report them during the interviews, especially as the conversation started with plants and no leading questions were subsequently asked.
Conducted interviews did not presume discussions about the healing properties of water, metal, fire or stones. The lack of comments pertaining to a number of folk magic rituals does not at all indicate their absence in the passive memory of the rural residents of the Liuban region or maybe even in their practical use; rather, it is not regarded as the type of knowledge to be willingly shared with a stranger, as is the use of plants for healing. As the use of non-plant remedies was quite recently considered superstition and the "remains of old times", not suitable for the new social reality, it may well be that people are not willing to open up and speak freely so as not to sound unknowledgable. However, as non-plant uses are quite numerous for the ethnoveterinary use area, and some people reported only the use of non-plant remedies in either human or veterinary medicine, this assumption may not be entirely accurate.

\section{Conclusions}

This study examined the use of cultivated plants and non-plant remedies in human and ethnoveterinary mecidine and also tried to understand the importance of these groups of remedies compared with wild plants. While there may be a little bias in favor of wild plants

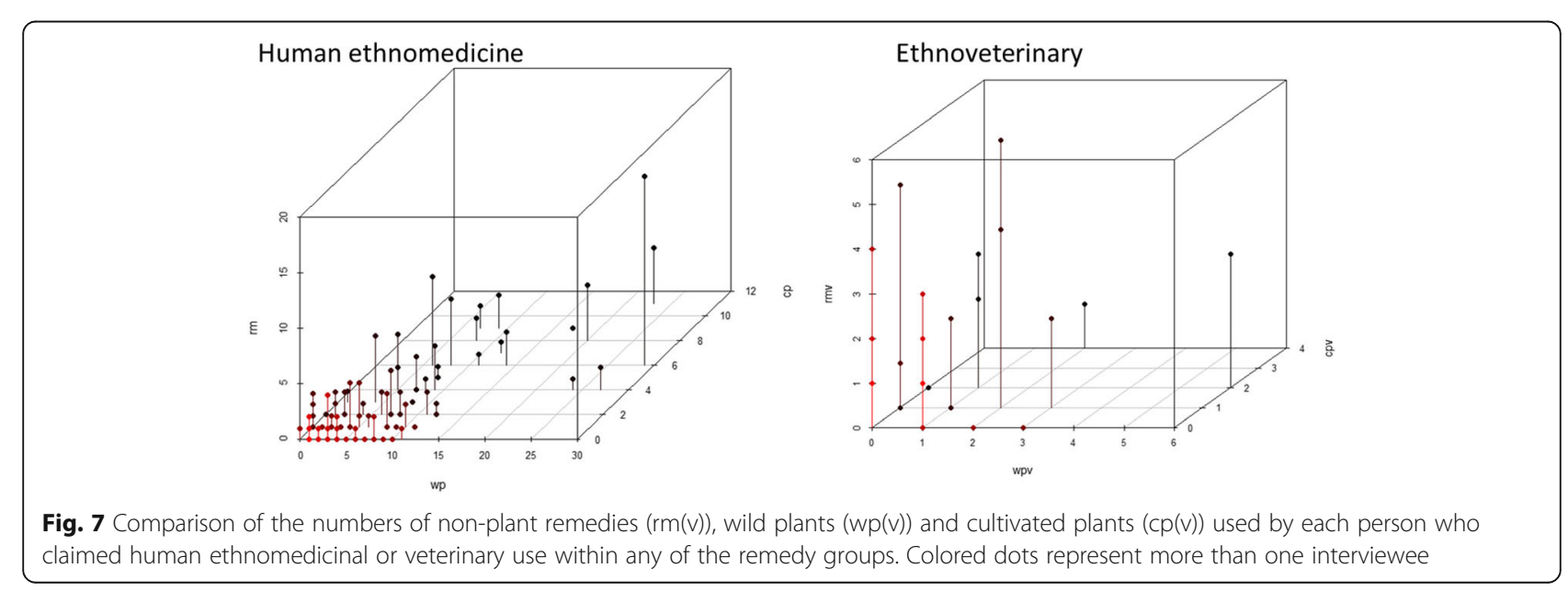




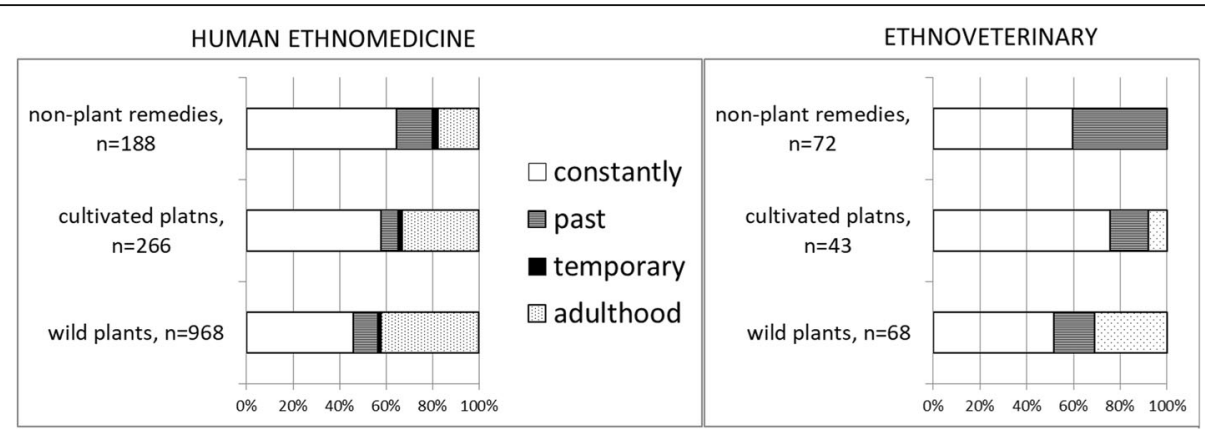

Fig. 8 Proportion of DUR in different temporal blocks in human ethnomedicine and ethnoveterinary medicine

due to the way information was collected (starting point of the interview was wild food plants), the differences among the three remedy groups were well pronounced, indicating that in domestic medicine cultivated plants and other means were remarkably less important than wild ones. In ethnoveterinary medicine non-plant remedies were nearly equally important as wild plants, while cultivated plants were the least utilized. However, being "at hand" does not necessarily mean being used or valued more, while remedies requiring more specific knowledge (such as wild-growing plants) are used more often and diversely. Even against the backdrop of the loss of unintentional contact with nature, people in Belarus more readily talk about using wild plants, supported by both official medicine and popular literature. While the use of non-plant remedies is somewhat promoted by popular literature, this alone does not seem to be sufficient to sustain local uses and/or open people up for sharing such uses. Therefore, even if our results indicate that proximity to the domestic arena does not influence the popularity of a remedy, we cannot say this with full certainty. Further research should develop more structured and detailed approaches and methods for evaluating the trends detected in the present study in order to better understand the mechanisms of the evolution of popular medicine.

\section{Abbreviations}

DUR: Detailed Use Records; FIC: Informant Consensus Factor; UC: Use Citations; UI: Use Instances

\section{Acknowledgements}

Our special thanks go to all the study participants of the communities in the Asovets village council who generously shared their knowledge. The authors thank Margus Kiis, Lagle Aim and Laura Rachel Vesik for assistance in the field.

\section{Funding}

The field study was a part of an ethnobotany field school financed by the Ministry of Foreign Affairs of Estonia through the Developmental Cooperation Program (projects 66-2015-A and 69-2016-A). Research was supported by an Estonian Science Foundation Grant IUT22-5 and by the European Union through the European Regional Development Fund (Center of Excellence in Estonian Studies, CEES).
Availability of data and materials

The voice-recorded interviews as well as their transcripts are stored at the The Center for Belarusian Culture, Language and Literature Research and are available for study by request. A structured and organized version of the data is available from the first author upon reasonable request.

\section{Authors' contributions}

RS developed the concept and drafted the manuscript. RS and RK designed the methodology and approach and also wrote the peer-reviewed project proposal that financed the field study. SV addressed organizational details during the fieldwork. All authors (except TV and AS) conducted field research. All authors (except RS, RK, MK, AS and TV) transcribed interviews and conducted initial systematization. RS and RK completed the data systematization and analyzed the data. YH, JPr, TV, IV, YK and AS contributed to the writing of the manuscript, as well as composing the historiography of the subject in Belarus and a description of the region. JPr and $\mathrm{YH}$ also contributed to the results section and discussion. JPa was responsible for verifying the correct phonetic spelling of local remedy names. All authors read and approved the final manuscript.

\section{Ethics approval and consent to participate}

As there is no special Ethics Committee for such research in Belarus, the ethical aspects of the study were approved by the The Center for Belarusian Culture, Language and Literature Research. The research adhered to the local traditions for such research and the Code of Ethics of the International Society of Ethnobiology [28]. Prior oral informed consent was obtained from all study participants.

\section{Consent for publication}

Not applicable.

\section{Competing interests}

The authors declare that they have no competing interests.

\section{Publisher's Note}

Springer Nature remains neutral with regard to jurisdictional claims in published maps and institutional affiliations.

\footnotetext{
Author details

'Department of Environmental Sciences, Informatics and Statistics, Università Ca' Foscari Venezia, via Torino 155, 30172 Mestre, VE, Italy. ${ }^{2}$ Estonian Literary Museum, Vanemuise 42, 51003 Tartu, Estonia. ${ }^{3}$ The Center for Belarusian Culture, Language and Literature Research, Surhanava St., 1, Bldg. 2, 220072 Minsk, Belarus. ${ }^{4}$ Valozhynski district, v. Vialikaya Dajnava, Padhornaya st., 118, 222352 Minsk Region, Belarus. ${ }^{5}$ Liubań District Culture Center, Pershamajskaya st., 30, 223820 Liubań, Belarus. ${ }^{6}$ The Belarusian State University of Culture and Arts, Rabkorauskaya st. 17, 220007 Minsk, Belarus. ${ }^{7}$ Estonian University of Life Sciences, Institute of Agricultural and Environmental Sciences, Kreutzwaldi 5, 51014 Tartu, Estonia.
} 
Received: 28 April 2017 Accepted: 7 September 2017

Published online: 03 October 2017

\section{References}

1. Zuzak TJ, Boňková J, Careddu D, Garami M, Hadjipanayis A, Jazbec J, Merrick J, Miller J, Ozturk C, Persson IA, Petrova G. Use of complementary and alternative medicine by children in Europe: published data and expert perspectives. Complement Ther Med. 2013;21:S34-47.

2. Posadzki P, Watson LK, Alotaibi A, Ernst E. Prevalence of use of complementary and alternative medicine (CAM) by patients/consumers in the UK: systematic review of surveys. Clin Med. 2013;13(2):126-31.

3. Alarcón R, Pardo-de-Santayana M, Priestley C, Morales R, Heinrich M. Medicinal and local food plants in the south of Alava (Basque Country, Spain). J Ethnopharmacol. 2015;176:207-24.

4. Quave $\mathrm{CL}$, Pieroni A. A reservoir of ethnobotanical knowledge informs resilient food security and health strategies in the Balkans. Nat Plants. 2015; 1:14021.

5. Zamudio F, Kujawska M, Hilgert NI. Honey as medicinal and food resource. Comparison between polish and multiethnic settlements of the Atlantic forest, Misiones, Argentina. The open complementary medicine journal. Spec Issue: Med Ethnobiol. 2010;2:58-73.

6. Sõukand R, Pieroni A. The importance of a border: medical, veterinary, and wild food ethnobotany of the Hutsuls living on the Romanian and Ukrainian sides of Bukovina. J Ethnopharmacol. 2016;185:17-40.

7. Sõukand R, Kalle R. Change in medical plant use in Estonian ethnomedicine: a historical comparison between 1888 and 1994. J Ethnopharmacol. 2011; 135(2):251-60

8. Kujawska M, Klepacki P, Łuczaj Ł. Fischer's plants in folk beliefs and customs: a previously unknown contribution to the ethnobotany of the polishLithuanian-Belarusian borderland. J Ethnobiol Ethnomed. 2017:13:20.

9. Shklyarov AP. Redkiye ovoshchnyye, pryano-aromaticheskiye i lekarstvennyye rasteniya [Rare vegetable, spicy-aromatic and medicinal plants.]. Minsk: BelNII ovoshchevodstva; 1999.

10. Lipnitskiy SS. Fitoterapiya $\vee$ veterinarnoy meditsine [Phytotherapy in veterinary medicine]. Minsk: Belarus'; 2006.

11. Shirko TS. Apteka $v$ sadu i ogorode [Pharmacy in the fruit and vegetable gardens]. Minsk: Polymya; 1994.

12. Astaf'yev Vl. Ogorod na podokonnike [Vegetable garden on the windowsill]. Minsk: Universitetskaye; 1995

13. Sõukand R, Hrynevich Y, Vasilyeva I, Prakofjewa J, Vnukovich Y, Paciupa J, Hlushko A, Knureva Y, Litvinava Y, Vyskvarka S, Silivonchyk H, Paulava A, Kõiva M, Kalle R. Multi-functionality of the few: current and past uses of wild plants for food and healing in Liubań region, Belarus. J Ethnobiol Ethnomed. 2017;13:10.

14. Republic Belarus': Entsiklopediya: V 6 t. Vol. 4. In: Pashkov GP, etc. editors Minsk: BelEnB; 2007.

15. Hołubieŭ V, Doŭnar A. Sielskaja haspadarka. In: Vialikaje kniastva Litoŭskaje: Encykłapiedyja. Minsk: BiełEn; 2005. p. 77-80

16. Kapyski Z. Zemlyarobstva [Agriculture]. In: Gistoryja syalyanstvy Belarusi ad starazhytnastsi da 1861 [History of the peasantry of Belarus from antiquity to 1861]. Vol. 1. Minsk; 1997: 181-86.

17. Sierbaŭ IA. Vičynskija palanie: materyjalnaja kultura: etnahrafičny narys Biełaruskaha Palessia. Minsk: Biełar. fond kultury; 2005.

18. Shpilevskiy PM. Puteshestviye po Poles'yu i Belorusskomu krayu. Minsk: Polymia; 2004.

19. Batalin A. Ogorodnichestvo i sadovodstvo. In: Istoriko-statisticheskiy obzor promyshlennosti Rossii. Sel'skokhozyaystvennyye proizvedeniya. Ogorodnichestvo, sadovodstvo i domashniye zhivotnyye. Gornaya i solyanaya promyshlennost'. Sankt-Peterburg: Tipografiya A.S. Suvorina; 1883. p. 1-41.

20. Piatkievič Č. Rečyckaje Paliessie. Minsk: Bielaruski knihazbor; 2004.

21. Rytov MV. Ogorodnichestvo v Belorussii. Sovremennoye sostoyaniye nashego sortovedeniya. In: Dokhodnoye plodovodstvo. Kursy promyshlennogo plodovodstva i ogorodnichestva, chitannyye v raznykh punktakh Rossii. Moskva: M.I. Grell; 1891

22. Entsiklopedicheskiy slovar' [Encyclopedic Dictionary]. In: Andreyevskogo IYe, editor. Vol. 16: Konkord - Koyalovich. Sankt-Peterburg: F. Brokgauz, I. Yefron; 1895. p. 22-26.

23. Entsiklopedicheskiy slovar' [Encyclopedic Dictionary]. In: Andreyevskogopod IYe, editor. Vol. 41a: Yaytseprovod - V[izhitsa]. Sankt-Peterburg: F. Brokgauz, I. Yefron; 1904. p. 859-65.
24. Severgin VM. Zapiski puteshestviya po zapadnym provintsiyam Rossiyskogo gosudarstva, ili Mineralogicheskiye, khozyaystvennyye i drugiye primechaniya, uchinennyye vo vremya proyezda cherez onyye v $1802 \mathrm{~g}$ Sankt-Peterburg: Tipografiya Imperatorskoy akademii nauk; 1803.

25. Rossiya. Polnoye geograficheskoye opisaniye nashego otechestva : Nastol'naya i dorozhnaya kniga dlya russkikh lyudey: Verkhneye Podneprov'ye i Belorussiya: Smolenskaya, Mogilevskaya, Vitebskaya i Minskaya gub. Vol. 9. Sankt-Peterburg: Izdaniye A.F. Devriyena; 1905.

26. Kirkor A. Narodnyy trud. In: Zhivopisnaya Rossiya: Otechestvo nashe v yego zemel'nom, istoricheskom, plemennom, ekonomicheskom i bytovom znachenii. Vol. 3. Sankt-Peterburg: Tipografiya M.O. Vol'fa; 1882. p. 205-18.

27. Hudson C. Folk history and ethnohistory. Ethnohistory. 1966;13(1/2):52-70.

28. International Society of Ethnobiology. International Society of Ethnobiology Code of Ethics (with 2008 additions). http://ethnobiology.net/code-ofethics/. Accessed 23 Sept 2017.

29. Tutin T, Heywood V, Burges N, Valentine D, Walters S, Webb D. Flora Europaea. Cambridge, UK: University Press; 1964.

30. The Plant List. Version 1. 2010. Published on the Internet; http://www. theplantlist.org/. Accessed 1 June 2017.

31. Stevens PF. Angiosperm Phylogeny Website. Version 14, July 2017. http:// www.mobot.org/MOBOT/research/APweb/. Accessed 30 July 2017.

32. Sõukand R, Kalle R. Where does the border lie: locally grown plants used for making tea for recreation and/or healing, 1970s-1990s Estonia. J Ethnopharmacol. 2013;150:162-74.

33. Łuczaj Ł, Kujawska M. Botanists and their childhood memories: an underutilized expert source in ethnobotanical research. Bot J Lin Soc. 2012;168:334-43.

34. Kalle R, Sõukand R. Wild plants eaten in childhood: a retrospective of Estonia in the 1970s-1990s. Bot J Lin Soc 2013:172(2):239-53.

35. Trotter RT, Logan MH. Informant consensus: a new approach for identifying potentially effective medicinal plants. In: Etkin NL, editor. Plants in indigenous medicine and diet, behavioural approaches. New York: Redgrave Publishing Company; 1986. p. 91-112.

36. Johns T, Kokwaro JO, Kimanani EK. Herbal remedies of the Luo of Siaya District, Kenya: establishing quantitative criteria for consensus. Econ Bot. 1990;44:369-81.

37. Valodzina T. Narodnaya miedycyna: rytualna-mahičnaja praktyka. Minsk: Bielaruskaja navuka; 2007.

38. Shein P. Materialy dlya izucheniya byta i yazyka russkogo naseleniya SeveroZapadnogo kraya. Vol. 1. Sankt-Peterburg: Tipografiya Imperatorskoy Akademii Nauk; 1887

39. Shein P. Materialy dlya izucheniya byta i yazyka russkogo naseleniya SeveroZapadnogo kraya. Vol. III. Sankt-Peterburg: Tipografiya Imperatorskoy Akademii nauk; 1902.

40. Romanov YE. Belorusskiy sbornik. Vol. 5. Vitebsk: Tip-lit. G.A.Malkina; 1891.

41. Romanov, YE. Belorusskiy sbornik. Vol. 8. Vil'no: Tip. A.G.Syrkina; 1912.

42. Romanov YR. Iz oblasti belorusskoy narodnoy meditsiny In: Mogilevskaya starina. Vol. 3. Mohiliov: Tipohrafija Hubiernskoho pravlienija; 1903. p. 107-24

43. Nikiforovskiy N. Prostonarodnye primety i povier'ya, suyevernyye obryady i obychai, legendarnye skazaniya o licakh i miestakh Vitebskoy Belorussii. Vitebsk: Gubernskaja tipo-litografija; 1897.

44. Nikiforovskiy N. Osvyashchennyye predmety i otnosheniye k nim prostonarod'ya Vitebskoy Belorussii. Vitebsk: Tipo-Litograf M.B.Nejmana; 1903

45. Federowski M. Lud Białoruski na Rusi Litewskiej. Materiały do etnografii słowiańskiej zgromadzone w latach 1877-1891. In: Wiara, wierzenia i przesądy ludu z okolic Wołkowyska, Słonima, Lidy i Sokyłki. Vol. 1. Kraków: Akademia Umiejętności; 1897.

46. Demidovich P. Iz oblasti verovaniy i skazaniy belorusov. In: Etnograficheskoye obozreniye. Moscow: Tipografija A.A.Levenson; 1896.

47. Orzeszkowa E. Ludzie i kwiaty nad Niemnem. Wisła. 1888:2:1-15. 675-703; 1890;4:1-31; 1891;5:235-247.

48. Fedorowski M. Lud Białoruski na Rusi Litewskiej. Vol. I. Kraków; 1897.

49. Wereńko F. Przyczynek do lecznictwa ludowego. Materiały anrtopologicznoarcheologiczne i etnograficzne. Vol. 1. Kraków: 1896. p. 99-229.

50. Romanov Ye. Belorusskiy sbornik [Belarusian collection]. Vol. 5. Vitebsk; 1891.

51. Jeleńska E. Wieś Komarowicze w powiecie mozyrskim. Warszawa: J. Jeżyński; 1892.

52. Nikiforovskiy NYA. Prostonarodnyye primety i pover'ya, suyevernyye obryady i obychai, legendarnyye skazaniya o litsakh i mestakh [Common folk signs and beliefs, superstitious rituals and customs, legendary tales of persons and places]. Vitebsk: Gubernskaya Tipo-Litografiya; 1897. 
53. Dobrovol'skiy VK. Smolenskiy oblastnoy slovar' [Smolenskiy regional dictionary]. Smolensk: Tipografiya P.A. Silina; 1914.

54. Sheyn PV. Materialy dlya izucheniya byta i yazyka russkogo naseleniya Severo-Zapadnogo kraya [Materials for studying the life and language of the Russian population of the North-West Territory]. Vol. 3. Sankt-Peterburg: P.V. Sheyn; 1902.

55. Sieržputoŭski AK. Prymchi i zababony bielarusaǔ-paliešukoŭ [Prejudices and superstitions of Belarusians-paleshukou]. Minsk; 1930.

56. Romanov YeR. Belorusskiy sbornik [Belarusian collection]. Vol. 8. Vil'na; 1912.

57. Min'ko LI. Narodnaya meditsina Belorussii (Kratkiy istoricheskiy ocherk). Minsk: Nauka i tekhnika; 1969

58. Min'ko LI. Narodnaya meditsina i vred znakharstva. № 19. Minsk; 1962.

59. Polacki etnahrafičny zbornik. In: Lobač UA, editor. Issue 1: Narodnaja medycyna bielarusaŭ Padzvinnia. Vol. 1-2. Navapolack; 2006.

60. Graniszewska MA, Leśniewska HA, Mankiewicz-Malinowska AL, Galera HA. Rośliny użyteczne... Michała Fedorowskiego - dzieło odnalezione po 130 latach. Useful plants... by Michal Fedorowski - the work found after 130 years. Etnobiolog Polska. 2013;3:63-120.

61. Łuczaj Ł, Köhler P, Pirożnikow E, Graniszewska M, Pieroni A, Gervasi T. Wild edible plants of Belarus: from Rostafiński's questionnaire of 1883 to the present. J Ethnobiol Ethnomed. 2013;9:21.

62. Sieržputoŭski AK. Prymchi i zababony biełarusaŭ-palešukoŭ. Minsk: Univiersiteckaje vydaviectva; 1998.

63. Wereńko F. Przyczynek do lecznictwa ludowego. In: Materjały antropologiczno-archeologiczne i etnograficzne. Vol. 1. Kraków: Wyd-wo Komisyi Antropolog. Akad. Umiętności; 1896. p. 99-228.

64. Grebennikov YeA. Babushkiny retsepty lecheniya medom. Minsk: Popurri; 2012

65. Shemetkov MF, Shapiro DK, Danusevich IK. Produkty pchelovodstva i zdorov'ye cheloveka. Minsk: Uradzhay; 1987.

66. Neumyvakin I. Med: mify i real'nost'. Sankt-Peterburg: Dilya; 2005.

67. Sitsinskiy AA. Akusherskaya pomoshch' v Minskoy gubernii: issledovaniye doktora meditsiny A. A. Sitsinskogo. Sankt-Peterburg: Tipografiya P.P. Soykina; 1893

68. Dobrovol'skiy VN. Smolenskiy etnograficheskiy sbornik. Ch. 1. SanktPeterburg: Tipografiya Y.Yevdokimova; 1891.

69. Bahdanovič A. Pieražytki staražytnaha svietasuzirannia ŭ bielarusaŭ. Etnahrafičny narys. Hrodno: Hubernskaya Typohrafiya; 1895.

70. Zelenin DK. Opisaniye rukopisey Uchenogo Arkhiva Imperatorskogo Russkogo Geograficheskogo Obshchestva. Petrograd: Tipografiya AV. Orlova; 1914.

71. Krachkovskiy YF. Ocherki byta zapadno-russkogo krest'yanina. In: Vilenskiy sbornik. Vil'no: V. Kulin; 1869. p. 160-209.

72. Krachkovskiy YF. Byt zapadno-russkogo selyanina. Moskva: Izdaniye Imperatorskogo Obshchestva Istorii i Drevnostey Rossiyskikh pri Moskovskom Universitete, Universitetskaya tipografiya; 1874.

73. Vallejo JR, González JA. The use of the head louse as a remedy for jaundice in Spanish folk medicine: an overview. J Ethnobiol Ethnomed. 2013:9:52.

74. Usacheva V. Ispug. In: Tolstoi NI. editor. Slavyanskiye drevnosti: Etnolingvisticheskiy slovar': V 5 t. Vol. 2. Moscow: Mezhdunarodnyye otnosheniya; 1999. p. 424-26.

75. Valodzína T. Cela chalaveka: slova, míf, rytual. Minsk: Tekhnalogíya; 2009. p. 240-45.

76. Po YN. Minskoy gubernii. In: Sbornik svedeniy dlya izucheniya byta krest'yanskogo naseleniya Rossii. Vol. 1. Moskva; 1889. p. 213-21.

77. Szukiewicz W. Wierzenia i praktyki ludowe. Wisła. 1903;XVII(3(4)):432-44.

78. Lobac U. Tradycyjnyja liekavyja srodki raslinnaha pachodžannia ŭ bielarusaŭ Padzvinnia. In: Lobač UA editor. Polacki etnahrafičny zbornik. Vol. 2: Narodnaja proza bielarusaŭ Padzvinnia. U 2 č. Č. 1. Navapolack: PD; 2011. p. 26-30.

79. Fries CJ. Self-care and complementary and alternative medicine as care for the self: an embodied basis for distinction. Health Sociol Rev. 2013;22(1):37-51.

80. Garady í voskí Belarusí: entsyklapedyya. Vol.8. Mínskaya voblasts'. Kn.3. T.U. In: Byalova TU editor. Mínsk: Belarus. Entsykl. Ímyaya P. Broŭkí; 2012.

\section{Submit your next manuscript to BioMed Central and we will help you at every step:}

- We accept pre-submission inquiries

- Our selector tool helps you to find the most relevant journal

- We provide round the clock customer support

- Convenient online submission

- Thorough peer review

- Inclusion in PubMed and all major indexing services

- Maximum visibility for your research

Submit your manuscript at www.biomedcentral.com/submit
C Biomed Central 